\title{
Acides gras inhabituels des organismes marins : une illustration de la biodiversité moléculaire marine
}

\author{
Gilles BARNATHAN \\ Groupe mer, molécules, \\ santé (MMS), EA 2160, \\ Équipe lipides marins à activité biologique, \\ Faculté des sciences pharmaceutiques \\ et biologiques, Pôle mer et littoral, \\ Université de Nantes, \\ 1, rue Gaston-Veil, BP 53508, \\ 44035 Nantes cedex 01, France \\ <gilles.barnathan@univ-nantes.fr>
}

\section{Environnement marin et biodiversité moléculaire des lipides et des acides gras}

L'environnement marin représente incontestablement un trésor d'organismes générant pléthore de métabolites primaires et secondaires qui gagneraient à être davantage explorés (Kornprobst, 2010). Cet environnement est très diversifié et les organismes marins, tout particulièrement les plus primitifs d'entre eux, produisent une grande variété de lipides et d'acides gras (AG) (Ackman, 1989 ; Bergé et Barnathan, 2005 ; Kornprobst, 2010). Les AG sont ubiquistes dans la nature et constituent la part essentielle des triglycérides, des cires, des glycolipides et des phospholipides (PL). Ainsi, à côté des AG polyinsaturés classiques, incluant ceux des séries bien connues $n-6$ et $n-3$, $d^{\prime}$ autres AG naturels insaturés doivent être pris en compte dans les efforts de recherche. La recherche se poursuit et apporte en effet périodiquement son lot de nouveaux AG identifiés. C'est ainsi que les algues et les invertébrés marins ont fourni des AG avec des insaturations de type non malonique (c'est-à-dire avec des doubles liaisons séparées par plus d'un méthylène). De nouveaux AG mono-insaturés et ramifiés, des AG acétyléniques (halogénés ou non) ont été identifiés. D'autres structures $\mathrm{d}^{\prime} \mathrm{AG}$ ont été révélées, comportant une fonction oxygénée additionnelle (hydroxyle, méthoxyle, acétoxyle), un halogène (chlore, brome). Les AG insaturés conjugués n'ont pas

\begin{abstract}
In addition to the common polyunsaturated fatty acids (FA) such as those of the $n-3$ and $n-6$ series with major interest in health and nutrition, marine organisms (invertebrates and algae) produce unique FA including branched, methoxylated, halogenated, acetylenic and non-methylene-interrupted structures. The biosynthetic pathways, and the structural and functional roles of these uncommon FA are often not yet known. This short overview wishes to be an outline on the particular FA classes possessing uncommon structures, especially those of increasing interest regarding their promising biological activities.
\end{abstract}

Key words: acetylenic fatty acids, biological activity, branched monounsaturated fatty acids, halogenated fatty acids, marine organisms, methoxy fatty acids, non-methyleneinterrupted fatty acids

été rapportés chez les organismes marins bien qu'ils aient été identifiés chez des micromycètes d'origine marine et cultivés (Ruiz et al., 2007). Ils ne seront donc pas examinés dans cet article. De tels AG inhabituels ne peuvent provenir, pour une large part, que de voies de biosynthèse originales. Peu d'information est actuellement disponible sur ces AG inhabituels, sur leur possible rôle dans les membranes cellulaires, leur biogenèse, leur potentiel biologique et biomédical, en comparaison avec les propriétés des AG conventionnels.

Cette brève mise au point bibliographique se propose de mettre l'accent sur quelques exemples marquants d'AG encore peu connus sans viser l'exhaustivité : insaturés non classiques, mono-insaturés et ramifiés, acétyléniques, halogénés, méthoxylés.

\section{Désignations et notations conventionnelles des AG}

S'il est vrai que les plus fréquents des AG communs, saturés ou insaturés, comportent un nombre pair d'atomes de carbone, certains organismes marins contiennent des AG impairs en nombre et en quantité relative non négligeable. Les AG polyinsaturés les plus fréquents ont des chaînes jusqu'en $C_{22}$ et avec au plus six doubles liaisons de configuration cis séparées par un groupe méthylène (methylene- interrupted) (Ackman, 1989 ; Gunstone et al., 1994 ; Cuvelier et al., 2004 ; Christie, 2010).

Une nomenclature largement utilisée pour de tels AG désigne par la notation 24:6 n-3 un AG à 24 carbones et six doubles liaisons, la première se trouvant au niveau du troisième carbone en comptant à partir du méthyle terminal, les autres étant séparées de celle-ci par un groupe $\mathrm{CH}_{2}$. Pour ces AG classiques, les doubles liaisons sont en principe cis, mais il est souvent nécessaire de le spécifier. À côté de cette «notation des biochimistes et physiologistes » existe une nomenclature, celle des chimistes, qui permet de nommer avec précision toute structure $d^{\prime} A G$ en indiquant par un indice la position d'une insaturation, d'une ramification, d'une fonction, en partant de la fonction acide carboxylique - $\mathrm{COOH}$ qui porte le numéro 1. Ainsi, pour l'acide 24:6 n-3 (acide 6,9,12,15,18,21-tétracosahexaènoïque), les autres possibilités sont $6,9,12,15,18,21$ $24: 6$ ou $24: 6 \Delta 6,9,12,15,18,21$. Divers exemples de structures sont présentés sur la figure 1 . Un AG de type non malonique (mollusques et autres invertébrés) correspond à un acide dans lequel deux doubles liaisons consécutives sont séparées par plus d'un groupe $\mathrm{CH}_{2}$, par exemple 7,15-22:2 ou 22:2 n-7,15 ou encore $22: 2$ $\Delta 7,15$ une notation très utilisée. L'acide en $C_{18}$ méthoxylé en alpha de la fonction et comportant deux doubles liaisons cis entre C-5 et C-6, et C-9 et C-10 est noté 2-méthoxy-5(Z), 9(Z)-octadécadiènoïque (éponge). L'acide iso-ramifié à 19 carbones et une double liaison 
cis entre les positions $\mathrm{C}-13$ et $\mathrm{C}-14$ sera noté 17-méthyl-13(Z)-octadécènoïque (éponge). L'acide 12-méthyloctadéca-17-ène-5-ynoïque (éponge) comporte une chaîne en $\mathrm{C}_{18}$, une double liaison en $\mathrm{C}-17$, une triple liaison en $\mathrm{C}-5$ et un méthyle en $\mathrm{C}-12$.

\section{Accès aux AG et à leurs dérivés : analyse et identification}

Les lipides totaux s'obtiennent habituellement à partir des algues ou des invertébrés marins au moyen d'une extraction par des mélanges de solvants polaires et non polaires. L'extraction peut concerner le corps entier d'un animal ou bien des parties ou tissus sélectionnés. L'extrait brut peut être soumis à une saponification qui sépare les insaponifiables et permet d'obtenir les AG totaux. II peut de plus être soumis à une chromatographie préparative permettant d'obtenir successivement lipides neutres, glycolipides puis PL. La composition en AG de chaque classe de lipides pourra être déterminée. Les méthodes d'extraction et de séparation, ainsi que les méthodes d'analyse structurale, sont en particulier présentées dans des sites Internet régulièrement mis à jour et dans les références proposées (Christie, 2010 ; Leray, 2010).

Les organismes marins contiennent des mélanges complexes d'AG, et la méthode analytique de choix reste le couplage chromatographie en phase gazeuse - spectrométrie de masse (CPG-SM). L'analyse basique s'adresse aux esters méthyliques, dérivés volatils produisant de nombreux ions fragments diagnostiques après impact électronique (figure 2).

Néanmoins, les esters méthyliques ne permettent pas, le plus souvent, de déterminer avec précision la position d'une double ou d'une triple liaison, d'une ramification, d'une fonction supplémentaire. II faut alors recourir à un autre type de dérivé contenant un atome d'azote (Christie, 2010), principalement $\mathrm{N}$-acyl pyrrolidide (Andersson, 1978), diméthyloxazoline (Dobson et Christie, 2002), ester picolynique (Harvey, 1992) (figure 2).

Afin d'observer et de caractériser des AG minoritaires, par exemple non maloniques, il peut s'avérer utile de préparer des fractions $d^{\prime} A G$ diéniques ou polyéniques au moyen d'une chromatographie préparative sur gel de silice imprégné de nitrate d'argent (Christie, 2010 ; Leray, 2010). Il existe aussi plusieurs procédés pour sécuriser la détermination de la position des insaturations en préparant par exemple des dérivés d'addition tels que les adduits diméthyldisulfures (Christie, 2010; Leray, 2010).
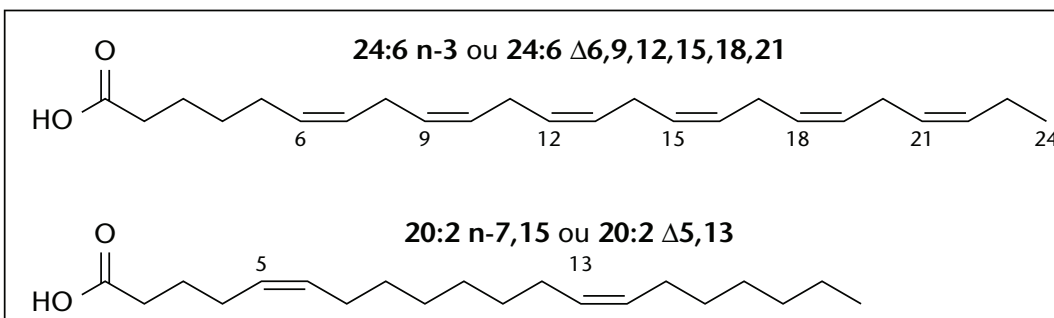<smiles>[Z20]CCCCC/C=C\CCCCCC/C=C\CCCCCC(=O)O</smiles><smiles>CCCCCCCC/C=C\CC/C=C/CCC(C)C(=O)O</smiles><smiles>CCCCCCCCCCCCCCCC/C=C/CCC(Br)=CCCCC(=O)O</smiles><smiles>CC(C)CC/C=C/CCCCCCCCCCCC(=O)O</smiles>

12-méthyloctadéca-17-ène-5-ynoïque<smiles>C=CCCCCC(C)CCCCCC#CCCCC(=O)O</smiles>

Figure 1. Structures d'acides gras marins inhabituels et nomenclature.

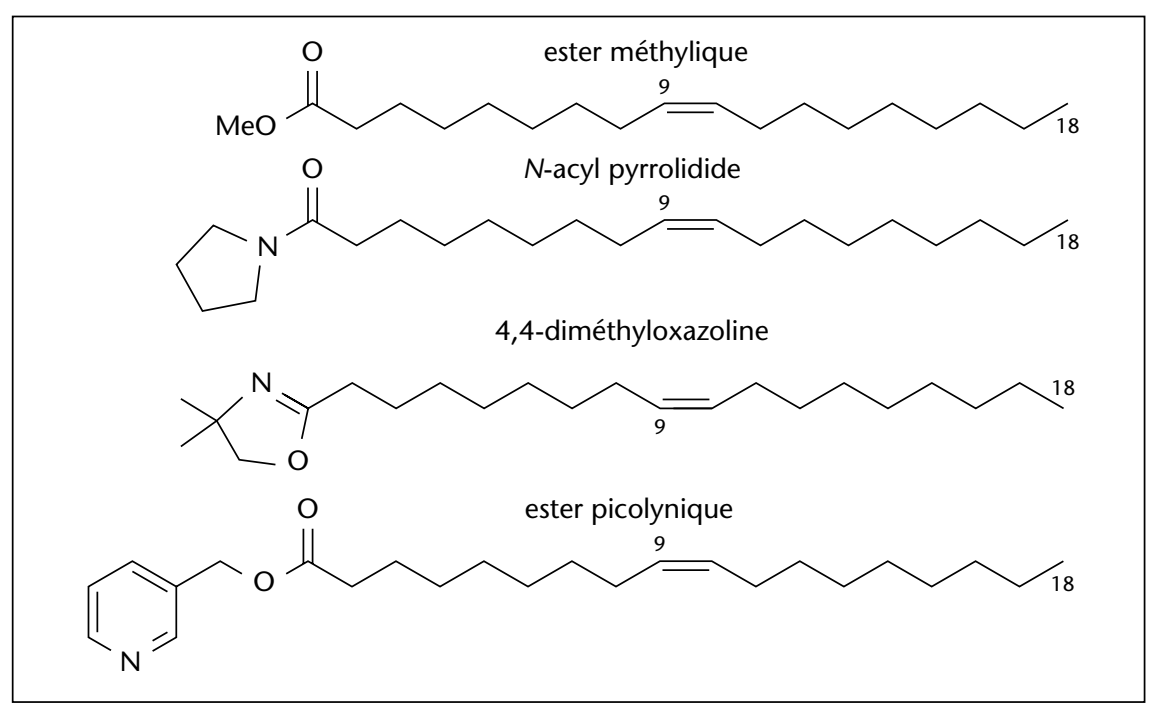

Figure 2. Dérivés volatils d'acides gras pour l'analyse par couplage CPG-SM, exemple de I'acide oléique. 


\section{AG polyinsaturés maloniques à très longue chaîne}

Avant de traiter des AG de structures inhabituelles, il semble intéressant de s'arrêter au cas des AG conventionnels, de type malonique, des séries n-3 et n-6 (20:5 n-3, EPA ; 22:6n-3, DHA; arachidonique 20:4 n-6), mais qui possèdent un nombre $d$ 'atomes de carbone supérieur à 22. De tels AG polyinsaturés ont été identifiés chez des organismes peu évolués: bactéries, microalgues, éponges, cnidaires. Une étude ancienne rapporte l'identification des acides 30:4 n-6 et 30:5 n-3 dans les lipides totaux et dans les PL de l'éponge Cliona celata (Litchfield et al., 1979).

Chez deux espèces de Gymnodinium et plusieurs autres dinoflagellés ont été détectés les AG inhabituels 28:7 n-6 et 28:8 n-3 à plus de $2 \%$ du total des AG (Mansour et al., 1999a). Les AG rares 28:7 n-3 et 28:7 n-6 ont été observés dans le hareng de la Baltique (Mansour et al., 1999b). Des teneurs significatives des acides 24:5 n-6 et 24:6 n-3 (jusqu'à $14 \%$ ) ont été relevées chez les échinodermes et les gorgones. Les spécimens de gorgones, collectés dans les eaux froides du littoral sénégalais après l'arrivée des alizés, contenaient des taux élevés d'AG polyinsaturés n-3 et n- 6 à l'opposé des taux très faibles constatés durant les périodes chaudes, en particulier 24:6 n-3 (5\%), 24:5 n-6 $(8,4-15,8 \%)$ et $24: 5$ n-3 (5\%) (Mirallès et al., 1995) (figure 1). Quelques cnidaires étaient distingués pour leurs teneurs élevées en acides 24:6 n-3 et 24:5 n-6 trouvés dans différents ordres des Octacoralliaires (Vysotskii et Svetashev, 1991), de plusieurs gorgones des genres Pseudopterogorgia et Eunicea et de la famille des Gorgoniidae (Carballeira et al., 1996; Carballeira et al., 1997a ; Carballeira et al., 2002). L'acide 24:6 n-3 a été identifié chez des échinodermes comme les ophiures et les holothuries (2-10\%) (Takagi et al., 1986; Kawasaki et al., 2000 ; Mansour et al., 2005). Une analyse des AG de la méduse australienne Aurelia sp. a révélé une présence majeure d'AG polyinsaturés (47,6\%), principalement arachidonique, EPA et DHA, mais aussi 24:6 n-3 $(9,3 \%)$ et $24: 5$ n-6 (0,8\%) (Nichols et al., 2003). Dans les PL de l'éponge Petrosia pellasarca, I'acide 34:5 n-3 a été identifié, pour la première fois dans l'environnement marin (Carballeira et Reyes, 1990a). La plupart des compositions précédentes incluent, comme AG insaturés majeurs, les acides 18:3 n-6, 18:4 n-3, arachidonique, EPA et DHA.

Les rôles et les éventuelles activités biologiques de ces AG polyinsaturés à très longue chaîne ne sont pas connus à l'exception du 24:6n-3, isolé de l'échinoderme Ophiura sarsi, pour lequel ont été mises en évidence des propriétés anti-inflammatoires et antiallergiques similaires à celles du DHA (Ishihara et al., 1998 ; Sato et al., 2001).

Un domaine de recherche nouveau a émergé à partir des années 1980 à la suite de la découverte de la faune des eaux profondes des sources hydrothermales et d'autres milieux extrêmes, et des nombreuses espèces animales entièrement originales qui $y$ prospèrent. Des publications en révèlent toute l'originalité en matière de lipides ainsi que l'a montrée une mise au point récente (Barnathan, 2009). À titre d'exemple, deux espèces de moules collectées entre 900 et $1200 \mathrm{~m}$ de profondeur en mer de Chine ou dans le Pacifique Nord ne comportaient pas les AG polyinsaturés $n-3$ et $n-6$, mais de nombreux $A G$ maloniques et cependant inhabituels des séries n-4 et n-7 : par exemple 18:3 n-7 ; 18:4 n-4 ; 20:3 n-7; 20:4 n-4 et 21:4 n-7 (Saito, 2008) (figure 3).

\section{AG mono-insaturés et ramifiés}

L'existence de bactéries dans les tissus des invertébrés marins est un phénomène connu qui se traduit, dans le domaine des lipides, par la présence d'AG caractéristiques. Avant de traiter des AG mono-insaturés et ramifiés rares, il est utile de donner un aperçu sur $d^{\prime}$ autres structures d'AG attestant de l'association d'un organisme marin avec des bactéries. II s'agit en particulier d'AG saturés impairs ramifiés tels qu'iso- et anteiso-15:0, et iso- et anteiso-17:0 (Kaneda, 1977 et 1991). Le phénomène peut être d'importance quand on sait que, par exemple, chez certaines éponges marines, les bactéries hébergées peuvent représenter plus du tiers du volume total des tissus (Vacelet, 1975). D’autres AG peu répandus en sont aussi des illustrations, par exemple : iso- et anteiso-16:0, iso- et anteiso-18:0, 10-Me-16:0, 12-Me-18:0 (Taylor et Parkes, 1983). Des AG de ce type, encore inconnus dans la nature ou dans le milieu marin, peuvent être découverts à l'occasion de diverses études de composition en AG. L'acide 15-Me-18:0 a ainsi été décrit dans les PL de l'éponge africaine Trikentrion laeve (Barnathan et al., 1996). L'association d'un invertébré marin avec des bactéries, et surtout des bactéries marines, est par ailleurs marquée par des AG monoinsaturés assez peu fréquents à longueur de chaîne modérée, tels que les acides 11-17:1,

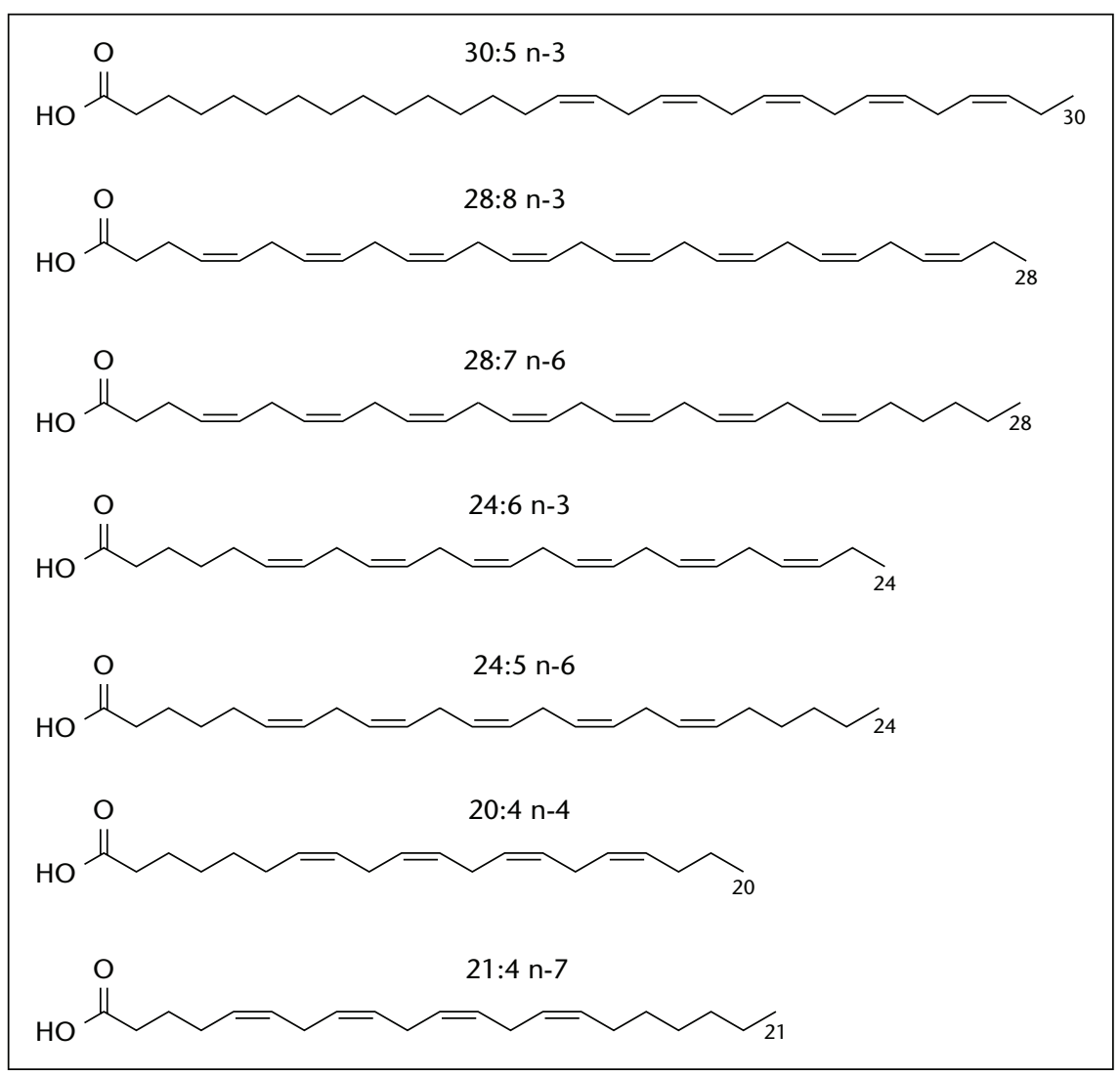

Figure 3. Exemples d'acides gras marins des séries n-3 et n-6 à chaîne $\geq C_{21}$. 
11-19:1 et 13-19:1. Ce dernier AG, plutôt rare, était présent à plus de $2 \%$ dans les $\mathrm{PL}$ de deux éponges Cinachyrella (Barnathan et al., 1992) et signait la présence de bactéries du genre Thiobacillus (Kerger et al., 1986).

L'association avec des bactéries ou avec $d^{\prime}$ 'autres micro-organismes s'avère particulièrement attestée par la présence des AG insaturés et ramifiés à chaîne relativement courte. L'étude de la composition en AG des lipides des invertébrés marins révèle souvent la présence d'AG inhabituels, voire originaux, monoènes à chaîne courte méthyl-ramifiée, par exemple en $\mathrm{C}_{15}-\mathrm{C}_{17}$. Ces $\mathrm{AG}$ sont considérés comme marqueurs bactériens (Boon et al., 1977; Boon et al., 1996). Ainsi, les acides iso-15:1 et iso-17:1 avec diverses positions de la double liaison, parfois rencontrés chez les invertébrés marins, sont caractéristiques de bactéries sulfatoréductrices telles que certaines espèces du genre Desulfovibrio (Dowling et al., 1988) (figure 4).

Les acides iso-4-pentadécènoïque, iso-5heptadécènoïque et anteiso-5-heptadécènoïque ont été par exemple identifiés dans les PL de l'éponge Polymastia penicillus, collectée en Bretagne à l'entrée du golfe du Morbihan (Denis et al., 2009). L'acide 15-méthyl-10(Z)-hexadécènoïque a été identifié dans plusieurs organismes, en particulier le mollusque siphonaride Siphonaria denticulata, et a été synthétisé ainsi que son homologue inférieur 13-méthyl-8(Z)-tétradécènoïque compte tenu de leur intérêt biologique (Carballeira et al., 2007b). Ces AG impairs, iso-ramifiés de type $n-6$ ont certainement une origine bactérienne à l'instar de leurs isomères $n-7$ qui ont été davantage étudiés (Kaneda, 1991). Cependant, de tels AG restent trop peu explorés du point de vue de leur intérêt éventuel comme agents thérapeutiques en dépit du fait que des AG iso-ramifiés similaires ont montré une importante activité inhibitrice de la topoisomérase-l et une activité cytotoxique (Lee et al., 1998 ; Reyes et Carballeira, 1996 ; Carballeira et al., 2007a). L'acide iso9-heptadécènoïque a été identifié dans des éponges et est attribuable à une bactérie marine (Boon et al., 1977 ; Walkup et al., 1981). Le nouvel acide 7-méthyl-8-hexadécènoïque et son isomère 14-méthyl-8-hexadécènoïque ont été identifiés dans l'éponge Desmapsama anchorata (Carballeira et Maldonado, 1988). Les deux stéréoisomères 7-méthyl-6(Z)-hexadécènoïque et 7-méthyl-6(E)-hexadécènoïque ont été observés chez $D$. anchorata (Carballeira et al., 1994a, b) (figure 4). Les gorgones Leptogorgia des côtes sénégalaises contenaient, en plus de taux élevés d'AG polyinsaturés en $C_{20}$ à $C_{24}$, plusieurs AG saturés ramifiés, I'acide 7-méthyl-6hexadécènoïque et des AG encore inconnus dans la nature : 10-méthyl-6-hexadécènoïque,

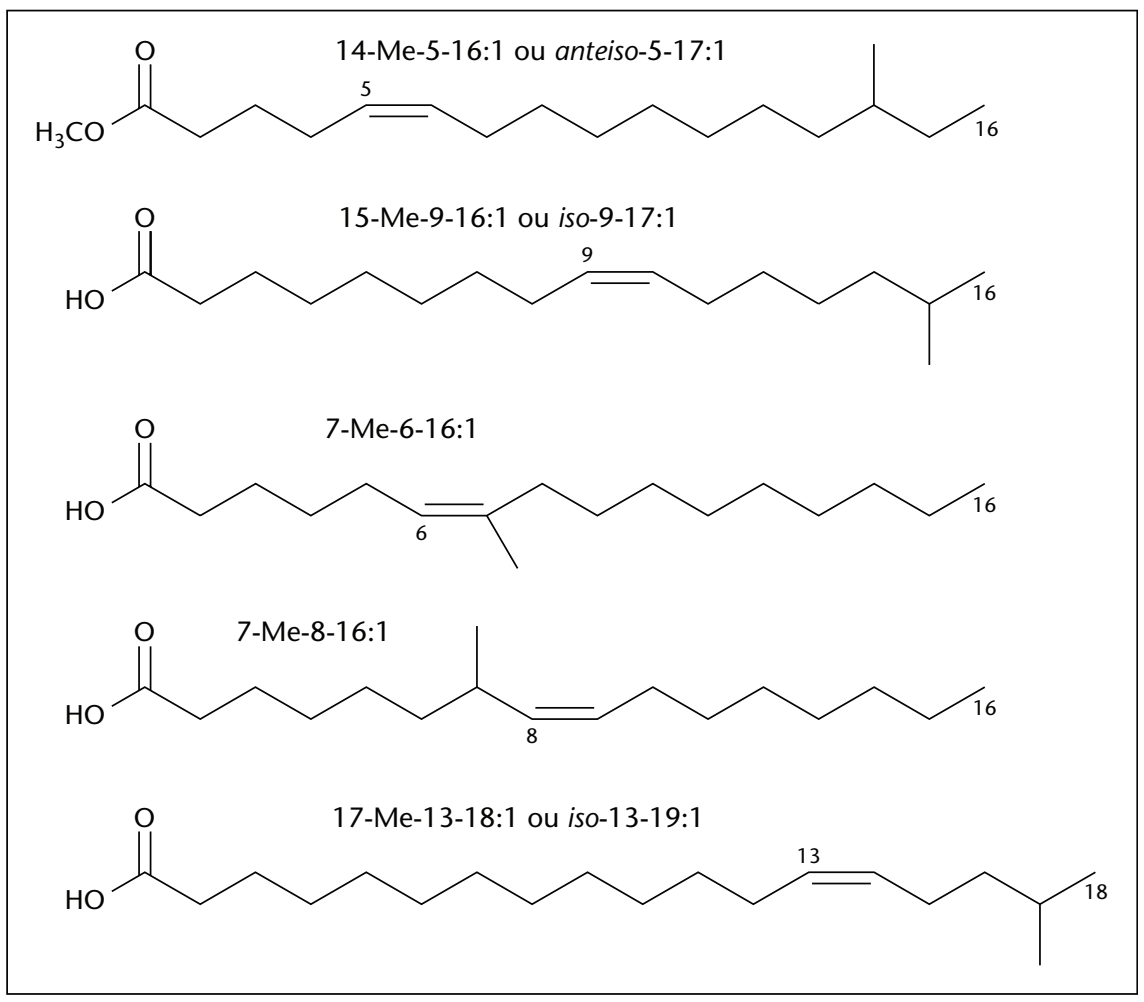

Figure 4. Exemples d'acides gras mono-insaturés ramifiés des organismes marins. 7,9-diméthyl-6-hexadécènoïque et 10-méthyl6,9-heptadécènoïque (Mirallès et al., 1995). L'acide original 7,8-diméthyl-7-hexadécènoïque a été observé, avec le 7-méthyl-7-hexadécènoïque, dans des huiles de poisson dans la fraction non complexée à l'urée (Ratnayake et al., 1989). Parmi ces AG monoènes ramifiés, il est opportun de mentionner les acides 17-méthyl$6(Z)$-octadécènoïque et 17-méthyl-7(Z)-octadécènoïque qui ont été isolés du mollusque gastéropode Siphonaria denticulata et préparés par synthèse compte tenu de leurs activités biologiques en particulier contre les parasites protozoaires (Carballeira et al., 2001). L'acide isomère 17-méthyl-11(Z)-octadécènoïque a été identifié dans l'éponge caribéenne Calyx podatypa (Carballeira et al., 1998). II est probable que ce dernier AG provienne en fait d'une bactérie marine puisqu'il a été primitivement caractérisé dans les bactéries sulfatoréductrices Desulfobacter et Desulfobulbus (Taylor, Parkes, 1983). Un autre isomère, le nouvel acide 17-méthyl-13(Z)-octadécènoïque a été récemment caractérisé chez $P$. penicillus (Denis et al., 2009). C'est l'acide iso-ramifié nonadécènoïque avec la double liaison la plus proche de l'extrémité de la chaîne, en n-5, et il a été obtenu par synthèse organique en raison de son activité contre le protozoaire Leishmania (Carballeira et al., 2009). Cette observation est renforcée par le fait que ce dernier AG semble provenir d'un allongement dicarboné de la chaîne de l'acide bactérien 15-méthyl-11(Z)-hexadécènoïque isolé de plusieurs sources, mais surtout chez Myxococcus xanthus (Boon et al., 1977 ; Reyes et Carballeira, 1996). II est intéressant de remarquer que $M$. xanthus est une mycobactérie dont les AG ont été signalés comme sensibilisant les cellules bactériennes Gram négatives et Gram positives à l'action des enzymes bactériolytiques (Boon et al., 1996).

\section{AG polyinsaturés non maloniques}

\section{AG non maloniques des mollusques}

Les travaux pionniers sur les AG non maloniques (non-methylene-interrupted) concernent surtout les mollusques (bivalves et gastéropodes) (Ackman et Hooper, 1973 ; Paradis et Ackman, 1975 et, 1977 ; Joseph et Fender, 1977 ; Johns et al., 1980 ; Ackman, 1989 ; Barnathan, 2009). On peut citer parmi ces travaux anciens la détermination de la composition en AG de divers tissus du mollusque bivalve Mercenaria mercenaria où les AG non maloniques se trouvaient en concentrations élevées dans les lipides polaires (13-19\%) (Klingensmith, 1982). 
Plusieurs acides di-insaturés de ce type, en $\mathrm{C}_{20}$ et $C_{22}$, ont été détectés : le plus abondant 22:2 $\Delta 7,15(22: 2 \mathrm{n}-7,15)$, à une moindre mesure son isomère 22:2 n-9,15 et, comme composants mineurs, les acides 20:2 $\Delta 7,13,20: 2$ $\Delta 7,11$ et $20: 2 \Delta 5,11$. Les auteurs remarquaient une intéressante relation inversée entre le niveau des $\mathrm{AG}$ dièniques non maloniques 22:2 et celui des AG polyinsaturés majeurs EPA et DHA (Klingensmith, 1982). Le bivalve Megangulus zyonoensis, une ressource alimentaire d'intérêt au Japon, et l'espèce $M$. venulosus contenaient les $A G$ diènoïques typiques de mollusques 22:2 $\Delta 7,13$ et 22:2 $\Delta 7,15$ (Kawashima et Ohnishi, 2003). La fraction diénique a été obtenue concentrée par chromatographie argentique, et huit acides non maloniques ont pu être identifiés: 18:2 $\Delta 5,11,20: 2 \Delta 5,11$, 20:2 $\Delta 5,13,20: 2 \Delta 7,13,21: 2 \Delta 7,13,21: 2$ $\Delta 7,15,22: 2 \Delta 7,13$ et $22: 2 \Delta 7,15$, dont trois observés pour la première fois chez les bivalves (Kawashima et Ohnishi, 2004). Les acides triinsaturés $20: 3 \Delta 5,11,14$ et $22: 3 \Delta 7,13,16$ étaient également présents (figure 5). Dans cette dernière étude, les autres AG insaturés majeurs étaient 18:1 n-9, 20:1 n-9, 20:1 n-7, 18:2 n-6, 18:2 n-4, 20:2 n-6, certains d'entre eux étant considérés comme intermédiaires biosynthétiques des AG non maloniques. Un travail portant sur plusieurs espèces de bivalves marins a montré une grande variation dans le contenu en $\mathrm{AG}$ non maloniques diènoïques : de 0,7 à $20,7 \%$ avec les acides principaux $22: 2 \Delta 7,13$ et 22:2 $\Delta 7,15$ (Zhukova et Svetashev, 1986). Plusieurs publications sur les lipides de mollusques (Trigari et al., 2001), de moules des genres Mytilus et Perna rapportent la présence des AG non maloniques (Fang et al., 1993 ; Zhakartsev et al., 1998 ; Garrido et Medina, 2002 ; Murphy et al., 2002 ; Ventrella et al., 2008). Mais les acides triènoïques 20:3 $\Delta 5,11,14$ et $22: 3 \Delta 7,13,16$ n'ont été observés que dans quelques études sur la moule commune Mytilus galloprovincialis (Zhakartsev et al., 1998). Une analyse de la fraction diènoïque de $M$. galloprovincialis a permis de caractériser plusieurs $A G$ insaturés non maloniques: $20: 2 \Delta 5,11 ; 20: 2 \Delta 5,13 ; 22: 2$ $\Delta 7,15$ et le nouvel acide $16: 2 \Delta 7,13$ (Garrido, Medina, 2002).

Les rôles et les propriétés biologiques de ces AG restent à explorer. II a été démontré par marquage isotopique que les bivalves sont capables de biosynthétiser ces $A G$ non maloniques, à la différence des AG polyinsaturés $n-3$ et $n-6$. II s'agit d'allongements par unités dicarbonées et de désaturation $\Delta 5$ à partir des acides 18:1 n-9 et $16: 1$ n-7 qui sont abondants dans le phytoplancton, en particulier chez les diatomées; une étape ultérieure d'allongement convertit l'acide 20:2 $\Delta 5,11$ en 22:2 $\Delta 7,13$ et l'acide 20:2 $\Delta 5,13$ en 22:2 $\Delta 7,15$ (Zhukova,
1986, 1991 ; Barnathan, 2009). On a pu montrer qu'ils sont souvent concentrés sur des PL particuliers, les plasmalogènes (Kraffe et al., 2004), et aussi qu'ils sont présents surtout dans les tissus exposés à l'environnement. Ces constats suggèrent un rôle de protection en particulier vis-à-vis de l'oxydation et des lipases microbiennes (Klingensmith, 1982; Fang et al., 1993 ; Kraffe et al., 2004 ; Barnathan, 2009).

\section{AG non maloniques des éponges}

Les éponges marines les plus primitifs des organismes multicellulaires ont représenté ces 40 dernières années une source exceptionnelle de métabolites et de lipides uniques: stérols, AG, PL, glycolipides (Djerassi et Lam, 1991 ; Bergé et Barnathan, 2005; Carballeira, 2008 ; Barnathan, 2009 ; Kornprobst, 2010). Ces invertébrés ne possèdent pas ou très peu

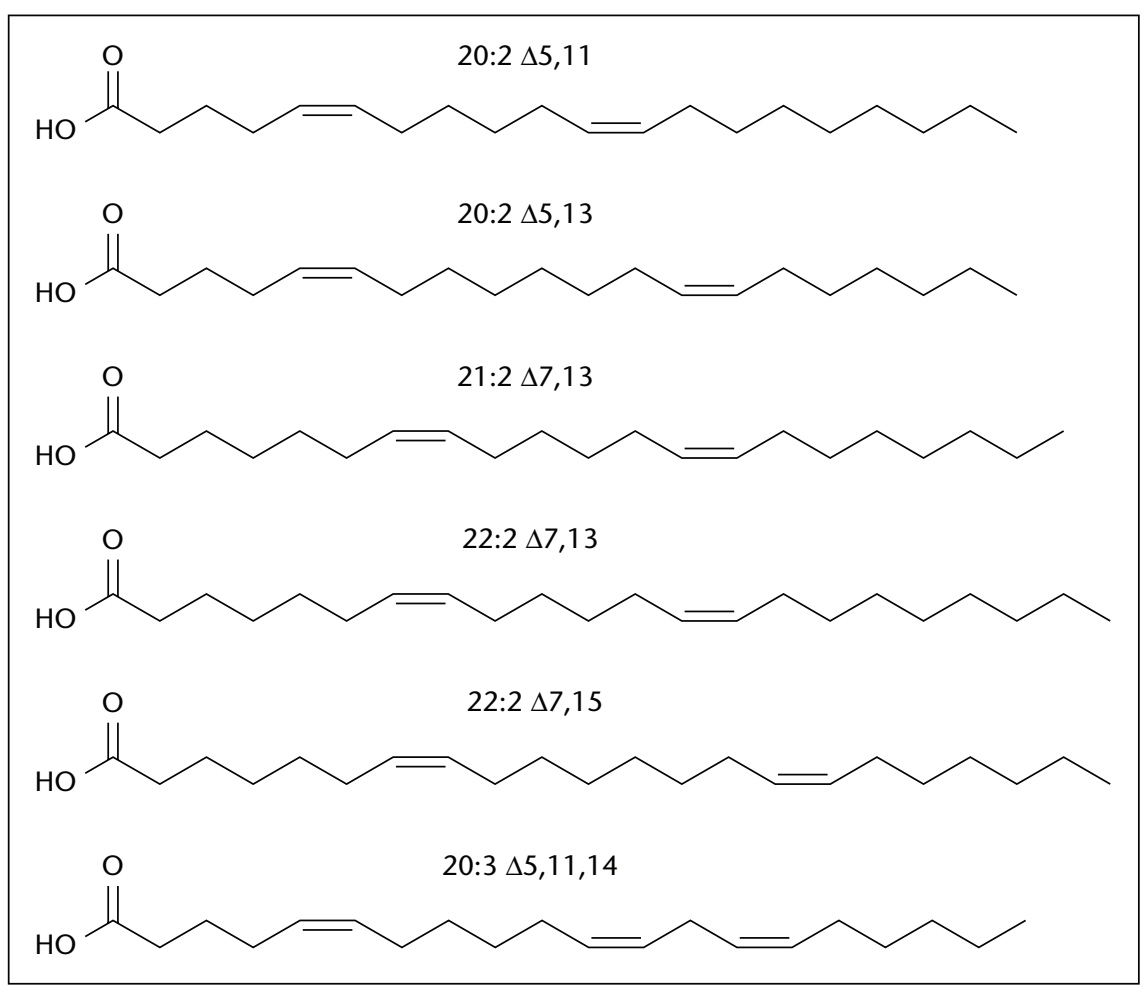

Figure 5. Acides gras non maloniques souvent présents chez les invertébrés marins.

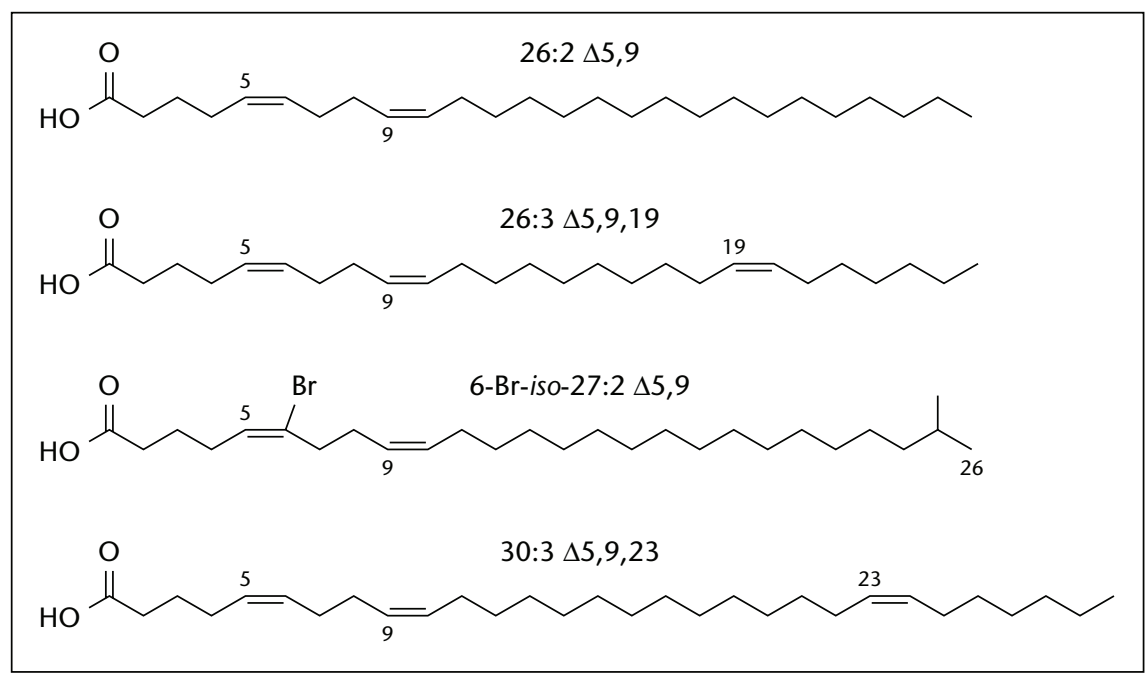

Figure 6. Exemples d'acides gras démospongiques $\Delta 5,9$ présents dans les éponges. 
d'AG polyinsaturés conventionnels mais des niveaux élevés d'AG à très longue chaîne $\left(>C_{22}\right)$ pairs et impairs, ramifiés ou non, parmi lesquels de nombreux AG originaux $y$ ont été identifiés.

\section{$A G$ à insaturation 45,9}

Les $A G$ à insaturation $\triangle 5 Z, 9 Z$ en $C_{24}$ à $C_{30}$ (mais aussi en $\mathrm{C}_{16}-\mathrm{C}_{22}$ ) se sont avérés les plus typiques de ces nouveaux AG. Une troisième insaturation est parfois présente vers l'extrémité hydrocarbonée de la chaîne acyle et aussi un atome de brome en C-6. De tels AG participent à la structure des membranes cellulaires. Parmi les plus fréquents, et parfois les plus abondants, on peut relever les acides $26: 2 \Delta 5,9 ; 26: 3$ $\Delta 5,9,19 ; 28: 3 \quad \Delta 5,9,21$ et $30: 3 \quad \Delta 5,9,23$ (Jefferts et al., 1974 ; Litchfield et al., 1979 ; Carballeira et Reyes, 1990a ; Djerassi et Lam, 1991 ; Barnathan et al., 1992) (figure 6). Certains nouveaux acides $\Delta 5,9$ possèdent une ramification méthyle ou un atome de brome en C-6 (Dasgupta et al., 1984 ; Carballeira et Reyes, 1990 b ; Barnathan et al., 1994 ; Barnathan et al., 2003 ; Nechev et al., 2004 ; Christie et al., 2004).

Les voies de biosynthèse des acides $\Delta 5,9$ des éponges ont été démontrées jusqu'aux structures en $C_{26}(26: 2 \Delta 5,19$ et $26: 3 \Delta 5,9,19)$ et comprennent des étapes d'allongement par unités dicarbonées et des étapes de désaturation à partir des précurseurs 16:0 et 16:1 n-7 (figure 7) (Morales et Litchfield, 1977 ; Djerassi et Lam, 1991). L'intermédiaire 26:0, ou bien l'intermédiaire 26:1 $\Delta 19$, est soumis à l'action soit d'une $\Delta 5$ désaturase, soit d'une $\Delta 6$ désaturase.

Les PL de certaines éponges peuvent posséder jusqu'à 15 acides $\Delta 5,9$, comme Trikentrion laeve, éponge des côtes sénégalaises (25,7 \% des AG des PL) (Barnathan et Kornprobst, 1992; Barnathan et al., 1996). En plus de l'acide $30: 3 \Delta 5,9,23(14,8 \%)$, trois autres $\mathrm{AG}$ en $\mathrm{C}_{30}$ y ont été découverts : 30:3 $\Delta 5,9,25(2.1 \%), 30: 1 \quad \Delta 23$ et $30: 2 \quad \Delta 9,23$ $(<1 \%)$ (figure 7). L'existence de ces deux derniers AG avait été postulée comme intermédiaires de biosynthèse du 30:3 $\Delta 5,9,23$ (Walkup et al., 1981). L'existence des acides $30: 3 \Delta 5,9,25$ et $26: 3 \Delta 5,9,21$ chez les éponges suggère une nouvelle voie de biosynthèse procédant à partir du 16:1 $\Delta 11$ par allongement en série monoènoïque $n-5$ (autre intermédiaire connu à l'état naturel, 24:1 $\Delta 17$ ) (Barnathan et Kornprobst, 1992 ; Barnathan et al., 1996).

\section{AG d'éponges non maloniques et non 45,9}

De nombreux autres AG insaturés non maloniques ont été par ailleurs identifiés dans les éponges, à commencer par ceux souvent rencontrés chez les mollusques, par exemple 20:2 $\Delta 5,11 ; 20: 2 \Delta 5,13 ; 22: 2 \Delta 7,13$ et $22: 2 \Delta 7,15$. De tels $A G$ ont été ainsi identifiés dans les éponges Dysidea fragilis (Christie et al., 1992),
Hymeniacidon sanguinea (Christie et al., 2004 ; Nechev et al., 2004), Amphimedon viridis (Carballeira et Shalabi, 1994a). L'acide 22:3 $\Delta 7,13,16$ a été observé chez Petrosia ficiformis (Ayanoglu et al., 1982).

Dans les PL de Euryspongia rosea ont été identifiés l'acide rare 18:2 $\Delta 6,11$ et le nouvel acide 20:2 $\Delta 6,11$ (Carballeira et Maldonado, 1989). Ce dernier AG a été trouvé dans les PL d'une autre éponge caribéenne qui contenaient aussi le nouvel acide 20:2 $\Delta 11,15$ (Carballeira et Restituyo, 1991). Plakortis halichondroides fait montre d'originalité en possédant des acides uniques avec des insaturations $\triangle 5 E, 9 E$ accompagnant le nouveau 20:2 $\triangle 6 E, 14 E$ (Carballeira et Shalabi, 1990c). L'éponge africaine Pseudaxinella cf. lunaecharta a permis d'identifier dans les PL deux nouveaux

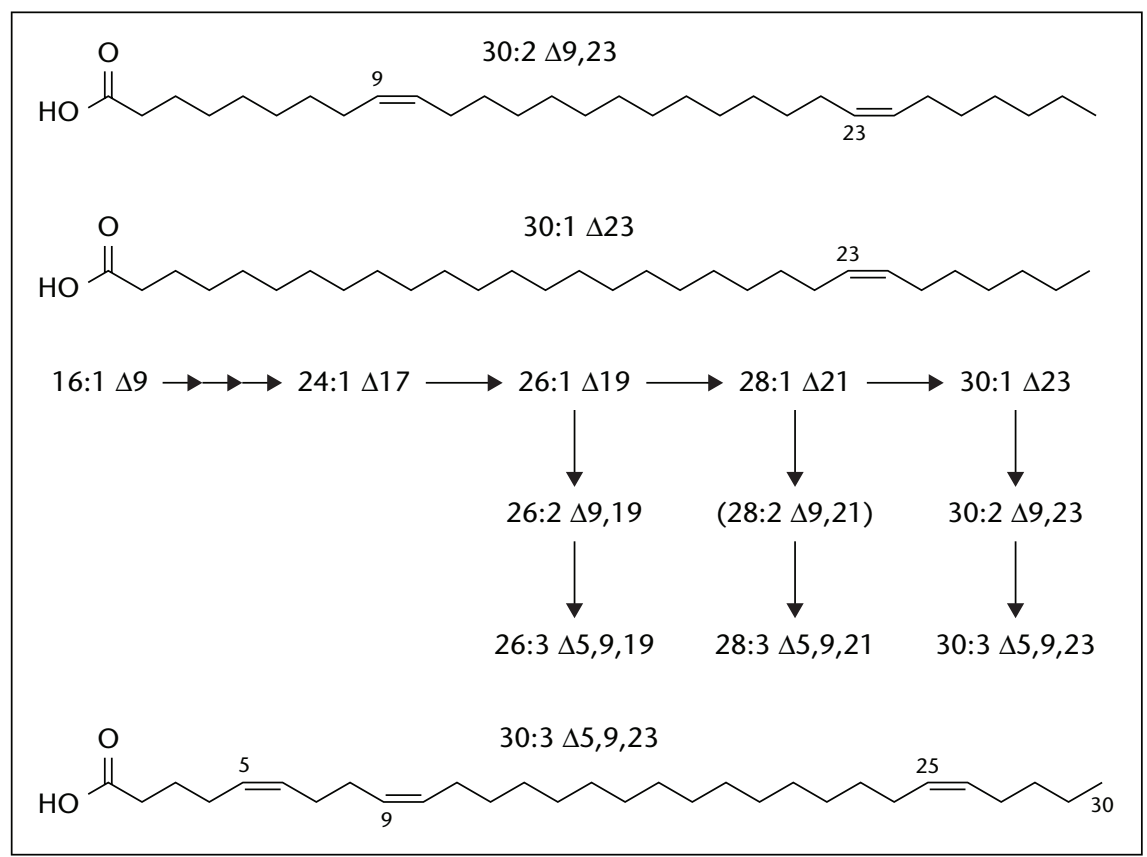

Figure 7. Exemples d'acides gras insaturés des éponges et rôle en intermédiaires de biosynthèse des acides gras $\Delta 5,9$.

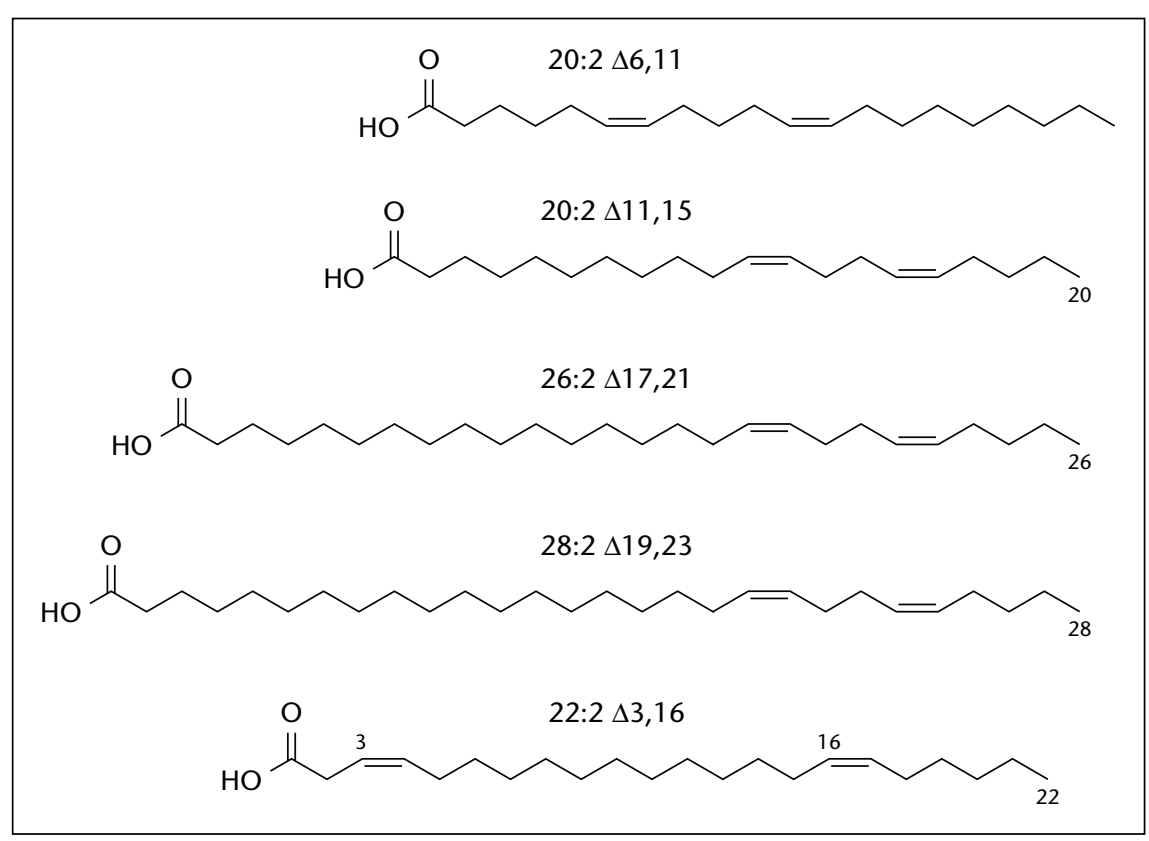

Figure 8. Quelques acides gras non maloniques et non $\Delta 5,9$ des éponges. 
AG - 26:2 $\Delta 17,21$ et 28:2 $\Delta 19,23$ - sans doute liés entre eux, et avec le 20:2 $\Delta 11,15$ cidessus, par une voie de biosynthèse partant d'un hypothétique 14:2 $\Delta 5,9$ inconnu à l'état naturel (Barnathan et al., 1996) (figure 8). Un AG non malonique original avec deux doubles liaisons situées vers chacune des extrémités de la molécule, l'acide 3,16-docosadiénoïque, a été détecté dans les PL de Polymastia penicillus (Denis et al., 2009) (figure 8).

\section{$A G$ non maloniques d'autres organismes}

Les PL des ophiures possèdent des taux élevés de 20:5 n-3 et 24:6 n-3 comme Ophiura sarsi (Ishihara et al., 1998 ; Kawasaki et al., 2000 ; Mansour et al., 2005), mais une série de nouveaux $\mathrm{AG}$ à doubles liaisons non maloniques cis et trans a été identifiée lors d'une étude ultérieure $d^{\prime} O$. sarsi (Sato et al., 2001): acides 7E,13E-20:2 ; 7E,13E,17Z-20:3 ; 9E,15E,19Z$22: 3 ; 4 Z, 9 E, 15 E, 19 Z-22: 4$ (figure 9). Les $A G$ non maloniques $20: 2 \Delta 7,13 ; 20: 2 \Delta 7,15$; $22: 2 \Delta 7,13 ; 22: 2 \Delta 7,13$ et le nouveau $22: 2$ $\Delta 6,14$ étaient présents dans les lipides totaux de divers organes trois raies de l'Atlantique tropical (Ould El Kebir et al., 2003, 2007). Ces AG non maloniques n'avaient jamais été signalés chez les poissons et les raies, bien que ces dernières se nourrissent largement de mollusques et de crustacés benthiques (Barnathan, 2009).

\section{AG $\Delta 5,9$ présents chez d'autres espèces que les éponges}

Depuis le milieu des années 1990, le domaine $\mathrm{d}^{\prime}$ existence des AG $\Delta 5,9$ s'est progressivement étendu aux anémones de mer (Carballeira et Medina, 1994b ; Carballeira et Reyes, 1995) et aux zoanthides, avec en particulier l'identification du nouveau 6-bromo-5,9-eicosadiènoïque (Carballeira et Reyes, 1995), aux gorgones (Carballeira et al., 1997b), aux mollusques (Kawashima et Onishi, 2004 et, 2008), aux nudibranches (Zhukova, 2007). Les AG non maloniques les plus fréquents chez les mollusques $(20: 2 \Delta 5,13$ ou $22: 2 \Delta 7,15$ par exemple) sont également souvent présents dans ces dernières compositions. Le nouvel 20:2 $\Delta 10,15$ a été trouvé parmi les AG libres d'un opistobranche de Méditerranée (Carballeira et al., 1992).

Ce n'est que récemment que des efforts de recherche importants ont montré un réel potentiel biomédical de ces AG $\Delta 5,9$ (Carballeira, 2008). Un mélange $C_{23}-C_{26}$ de ces acides a, par exemple, révélé une activité antipaludique prometteuse et une absence de cytotoxicité sur des cellules de mammifères (Tasdemir et al., 2007 ;
Carballeira, 2008). De plus, les acides $\Delta 5,9$ inhibent la topo-isomérase-I humaine (concentration inhibitrice $50 \%$ de 0,9-3 $\mu \mathrm{M}$ ) et sont actives contre des lignées cellulaires cancéreuses (Carballeira, 2008).

\section{AG acétyléniques}

Dans les AG acétyléniques des organismes marins, la triple liaison est souvent conjuguée avec une autre insaturation. Et il peut y avoir

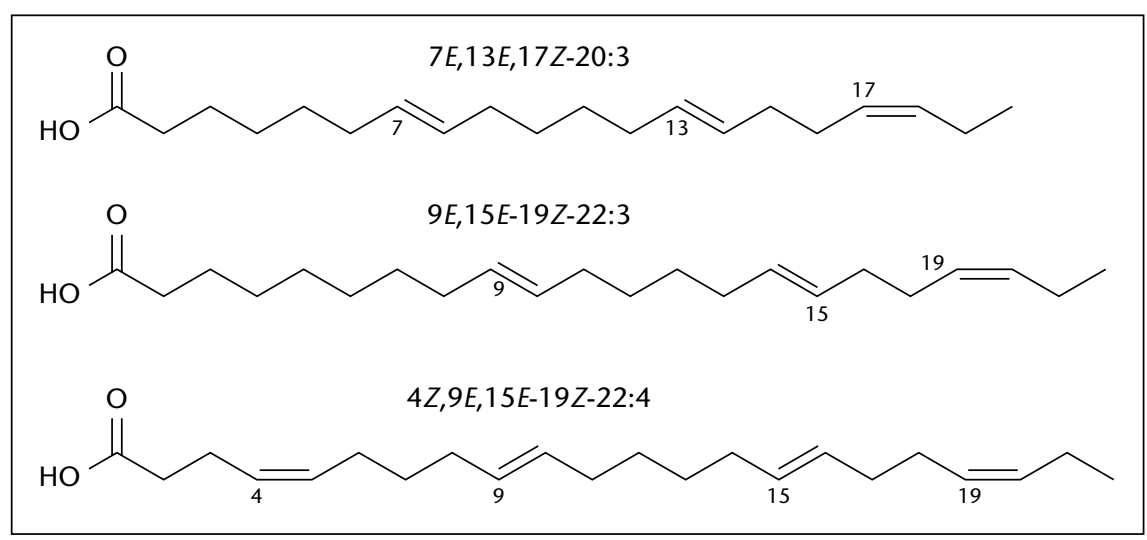

Figure 9. Acides gras remarquables des lipides d'une ophiure (échinoderme).

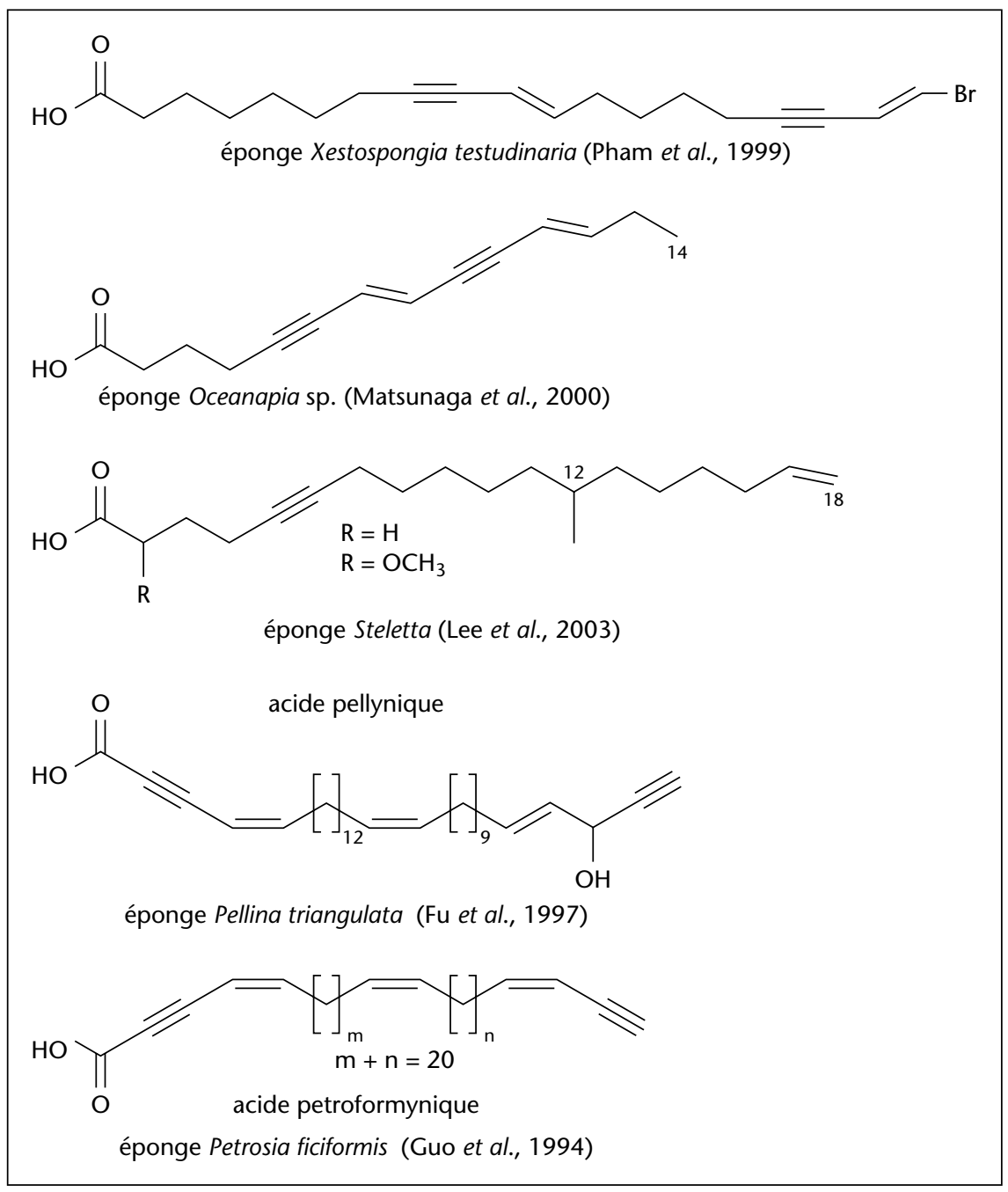

Figure 10. Acides gras acétyléniques des éponges. 
plus d'une triple liaison dans la formule d'un AG naturel de ce type. Un atome de brome, toujours en position vinylique, est souvent présent chez ces acides. Les chaînes des AG acétyléniques peuvent être ramifiées et, dans certains cas, méthoxylées.

Une première mise au point sur les AG acétyléniques naturels est parue en 1961 (Sörensen, 1961). Des AG acétyléniques ont été identifiés surtout chez les éponges. De tels AG, de masses moléculaires élevées et à activité biologique potentielle, ont été ainsi trouvés dans de nombreuses espèces d'éponges des genres Petrosia et Xestospongia (Quinn et Tucker, 1985 ; Hirsch et al., 1987 ; Dembitsky, 2006) (figure 10).

De tels AG, isolés de l'éponge Oceanapia sp., ont une activité significative contre des bactéries et des champignons, en particulier ceux à systèmes conjugués (Matsunaga et al., 2000). L'acide pétroformynique en $\mathrm{C}_{31}$ a été isolé de P. ficiformis (Guo et al., 1994). Plusieurs AG nouveaux avec une chaîne ramifiée et une partie acétylénique dans son milieu et une double liaison terminale ont été isolés par fractionnement bioguidé, chez des éponges du genre Steletta avec une cytoxicité modérée sur des lignées cellulaires cancéreuses humaines (Lee et al., 2003 ; Zhao et al., 2003). L'AG doublement acétylénique conjugué et bromé, présenté sur la figure 10, a été isolé par fractionnement bioguidé (affinité avec le récepteur A1 de l'adénosine) de l'éponge Xestospongia testudinaria (Pham et al., 1999). C'est un exemple de la grande variété des $\mathrm{AG}$ bromoacétyléniques (surtout en $C_{16}, C_{18}$ et $C_{20}$ ) qui ont été identifiés dans les éponges Xestospongia et Oceanapia (Van Soest et al., 1997). Les AG acétyléniques ramifiés, I'un $\alpha$-méthoxylé l'autre non, isolés d'une éponge Steletta, présentaient une activité modérée contre des lignées cellulaires de leucémie humaine (Lee et al., 2003). Les expérimentations par marquage isotopique menées de 1960 à 1990 ont permis d'établir que la majorité des AG acétyléniques dérivent de précurseurs $A G$ et polycétides (Minto et Blacklock, 2008). L'acide pellynique, avec d'autres composés acétyléniques, a été isolé de l'éponge Pellina triangulata et a présenté un effet d'inhibition de l'inosine monophosphatase déshydrogénase (figure 10) (Fu et al., 1997).

\section{AG halogénés}

De très nombreux AG halogénés, chlorés et bromés surtout, ont déjà été caractérisés chez une grande variété d'organismes marins, et il semble probable que leur nombre va nettement augmenter dans le futur. Une mise au point bibliographique sur les AG halogénés naturels a été publiée et traite, entre autres, de ceux des organismes marins (Dembitsky et Srebnic, 2002).

\section{AG chlorés}

Les AG chlorés ont été trouvés chez les poissons, les mollusques et autres invertébrés (Dembitsky et Srebnic, 2002). Les AG chlorés ont été d'abord identifiés dans un cnidaire, la méduse Auritia aurita. Six AG isomères, 9-Cl-10-OH-16:0 (9-chloro-10-hydroxypalmitique), $10-\mathrm{Cl}-9-\mathrm{OH}-16: 0, \quad 9-\mathrm{Cl}-10-\mathrm{OH}-18: 0$, 10-Cl-9-OH-18:0, $11-\mathrm{Cl}-12-\mathrm{OH}-18: 0$ et 12Cl-11-OH-16:0 y ont été identifiés pour la première fois dans cette classe d'invertébrés (White et Hager, 1977) (figure 11).

L'acide 9,10-dichloro-octadécanoïque a été isolé des lipides de $A$. aurita (White, Hager, 1977) et aussi de ceux de l'anguille Anguilla anguilla (Hakansson et al., 1991). Ce dernier AG, les acides 5,6-dichlorotétradécanoïque et 7,8-dichlorohexadécanoïque étaient présents sous les formes threo et erythro dans les lipides de plusieurs espèces de poissons comme l'anguille, le saumon, la perche, le hareng de la Baltique et de la mer du Nord (Mu et al., 1996, 1997). Les AG chlorés des lipides des bivalves ont été publiés (Wesen et al., 1995). Parmi les AG chlorés de la moule Mytilus edulis récoltée dans ces mêmes zones ont été découverts les acides 5,6dichoromyristique et 7,8-dichloropalmitique.

\section{AG bromés}

La présence de brome dans les substances marines est connue depuis les années 1930, et des concentrations de brome non négligeables ont été signalées dans les lipides de plusieurs animaux marins dans différentes zones maritimes, poissons, crustacés, mollusques, surtout dans les triglycérides et les AG (Tinsley et Lowry, 1980).

II revient au groupe de Wijekoon et al. d'avoir identifié les premiers $A G$ à insaturation $\Delta 5,9$ (souvent nommés « démospongiques» pour rappeler leur origine principale) porteurs $d^{\prime} u n$ atome de brome en position vinylique : acides 6-bromo-24-méthyl-5,9-hexacosadiènoïque (iso) et 6-bromo-25-méthyl-5,9-hexacosadiènoïque (anteiso) dans des éponges Petrosia (figure 11) (Wijekoon et al., 1984). Les acides 6-bromo-23-méthyltétracosa-5E,9Z-diènoïque et 6-bromo-24-méthylpentacosa-5E,9Z-diènoïque ont été identifiés chez Agelas sp. (Carballeira et Emiliano, 1993). En plus des éponges, d'autres invertébrés marins ont fourni des AG 6-bromo-5,9-diènoïques nouveaux ou rares : les anémones de mer (cnidaires) Condylactis gigantea (Carballeira et Reyes, 1995) et Stoichactis helianthus (Carballeira et Medina, 1994b), le zoanthide Palythoa caribaeorum (Hexacorallina) (Carballeira et Reyes, 1995). Des AG démospongiques bromés ont été aussi trouvés dans les éponges Amphimedon terpenensis (Garson et al., 1994), Hymeniacidonid sp.

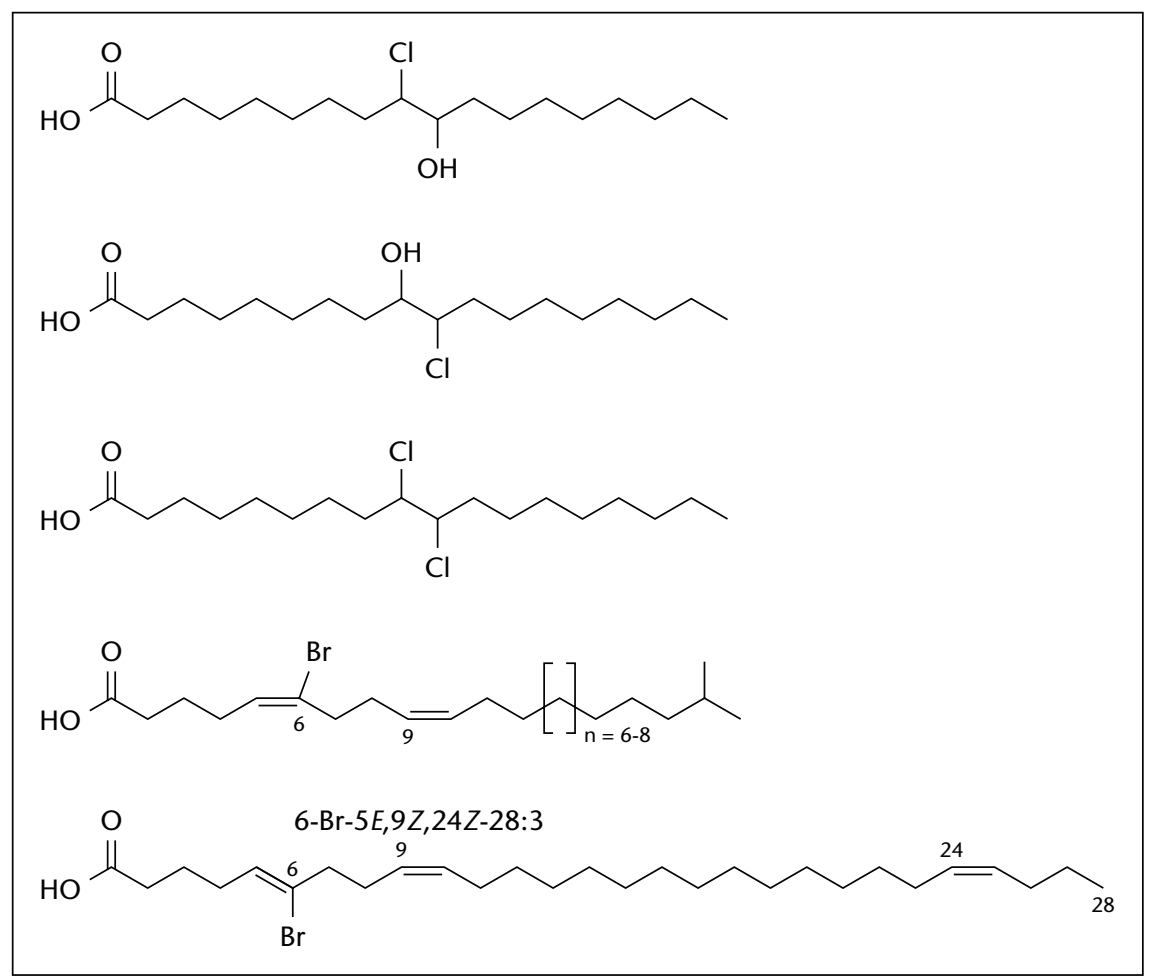

Figure 11. Acides gras halogénés des organismes marins. 
(Lam et al., 1989), Petrosia sp. (Carballeira et Shalabi, 1993 ; Carballeira, 1997b), Xestospongia sp. (Li et al., 1995), Cinachyrella aff. schulzei (Barnathan et al., 1994), Cinachyrella alloclada (Barnathan et al., 2003). L'acide 6bromo-5,9,13-docosatriènoïque a été identifié chez l'anémone de mer Stoichanthus helianthus (Carballeira et Medina, 1994b) et les acides 6-bromo-heptacosa-5E,9Z,24E-triènoïque et 6bromo-octacosa-5E,9Z,24Z-triènoïque d'éponges Xestospongia sp. (figure 11) (Li et al., 1995). Ces deux derniers AG présentaient une cytoxicité contre les cellules de leucémie murine L1210 et les cellules KB de carcinome épidermique humain (Li et al., 1995).

L'acide pétrosynilique inhibe la transcriptase inverse du virus d'immunodéficience humaine (Isaacs et al., 1993). Quelques AG polyacétyléniques bromés (en $\mathrm{C}_{18}$ en particulier) ont été identifiés dans diverses éponges (Dembitsky et Srebnik, 2002). Une série d'AG polyacétyléniques en $C_{23}$ bromés en position $C$-18, et leurs esters éthyliques, ont été découverts dans une éponge australienne Phakellia ; ces composés montraient une réaction positive contre des bactéries Gram positives (Dembitsky et Srebnik, 2002). Des AG acétyléniques en $C_{16}$ mono-, di- et tribromés ont été isolés d'éponges Xestospongia. Plusieurs AG acétyléniques ont été trouvés dans une Xestospongia sp. de mer Rouge (Hirsch et al., 1987). L'acide 14,16-dibromohexadéca-7E,13E,15Z-trièn-5ynoïque a été isolé d'une Xestospongia australienne (Schmitz et Gorichand, 1978). Les AG bromés des éponges Xestospongia et leurs esters ont une activité antibactérienne significative (Bourguet-Kondracki et al., 1992) et inhibent l'action de l'enzyme protéase jouant un rôle crucial dans la réplication du virus d'immunodéficience humaine (Patil et al., 1992). Les éponges des familles Nepheliospongia et Haploscleria produisent aussi des métabolites et des AG acétyléniques (Cimino et al., 1985). De nouveaux $A G$ acétyléniques bromés en $C_{18}$ tri- et diacétylèniques ont été identifiés dans les lipides d'une Petrosia en mer du Japon et étaient tous antifongiques contre des souches Mortierella pathogènes (Fusetani et al., 1993). De nombreux autres AG acétyléniques bromés ont été isolés d'éponges marines (Dembitsky et Srebnik, 2002).

\section{AG méthoxylés}

Les AG méthoxylés sont peu répandus dans la nature et restent limités aux organismes primitifs tels que cyanobactéries, bactéries et éponges (Kornprobst et Barnathan, 1998 ; Carballeira, 2002). Les premiers AG $\alpha$-méthoxylés naturels ont été trouvés dans les PL de l'éponge africaine Higginsia tethyoides: AG saturés, mono-insaturés et di-insaturés a-méthoxylés avec des chaînes de $C_{19}$ à $C_{21}$ (Ayanoglu et al., 1983a, b). Ces AG $\alpha$-méthoxylés qui possèdent un centre chiral de configuration $R$ ont été identifiés chez plusieurs autres éponges des genres Amphimedon, Callyspongia, Spheciospongia (figure 12) (Carballeira et Pagan, 2001 ; Carballeira et Colon, 1999).

Une mise au point a recensé 29 acides $\alpha$ méthoxylés naturels (Kornprobst et Barnathan, 1998). Les acides 2-OMe-5,9-18:2 et 2-OMe5,9-26:2 ont été identifiés chez les éponges. En plus des premiers AG $\alpha$-méthoxylés à chaîne $\mathrm{C}_{19}-\mathrm{C}_{28}$, plusieurs $\mathrm{AG}$ à courte chaîne $\left(C_{14}-C_{18}\right)$ ont été identifiés ensuite et ont été attribués à une probable présence de bactéries associées aux éponges. La méthoxylation en $\alpha$ confère à ces $A G$ une notable activité antifongique (Carballeira, 2002 ; Carballeira, 2008). Plusieurs AG méthoxylés en milieu de chaîne ont été identifiés seulement dans certains micro-organismes et cyanobactéries marines (genre Lyngbya) estérifiés à des métabolites biologiquement actifs, souvent chlorés, comme les malyngamides: 7-méthoxy-4dodécènoïque et 7-méthoxy-4-tétradécènoïque (figure 13) (Mesguiche et al., 1999 ; Kornprobst et Barnathan, 1998).

Les lipides de I'algue rouge Schyzimenia dubyi contenaient quatre nouveaux $\mathrm{AG}$ en milieu de chaîne (16\% des AG totaux) : 9-MeO-15:0, 9-MeO-17:0, 13-MeO-21:0 et 15-MeO-23:0 (Barnathan et al., 1998) (figure 13). La synthèse du 9-MeO-15:0 a été réalisée (Carballeira et Miranda, 2003).

\section{Conclusions et perspectives}

Cette revue des principaux aspects de la diversité moléculaire du monde marin sur le seul plan des $A G$ révèle cependant un gisement extraordinaire de potentialités biologiques et biomédicales. Néanmoins, on est encore bien loin d'en avoir exploré toutes les richesses s'agissant et de l'étendue des organismes sujets de ces efforts de recherche et de la compréhension des voies de biosynthèse, des rôles et

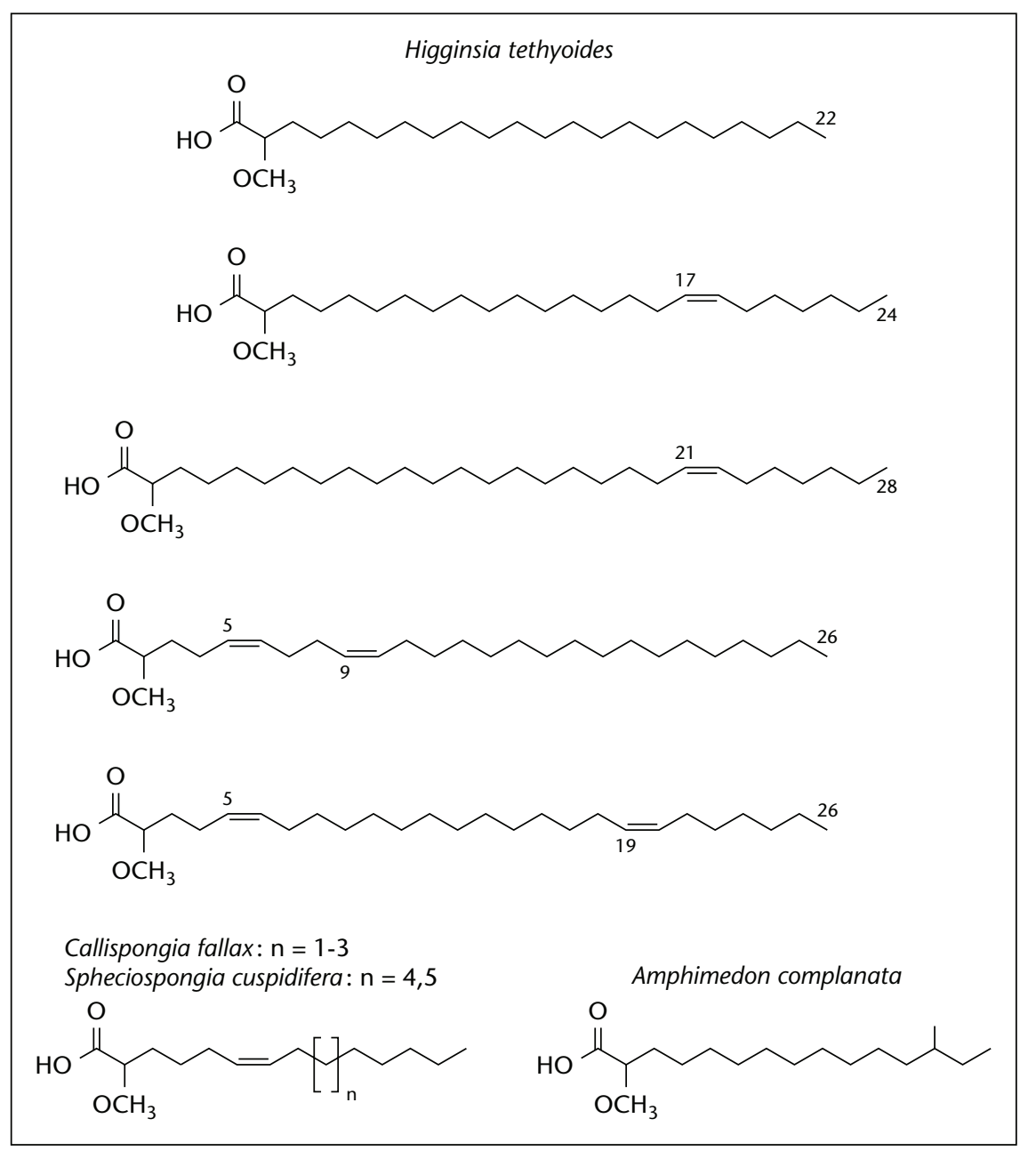

Figure 12. Acides gras $\alpha$-méthoxylés des éponges. 


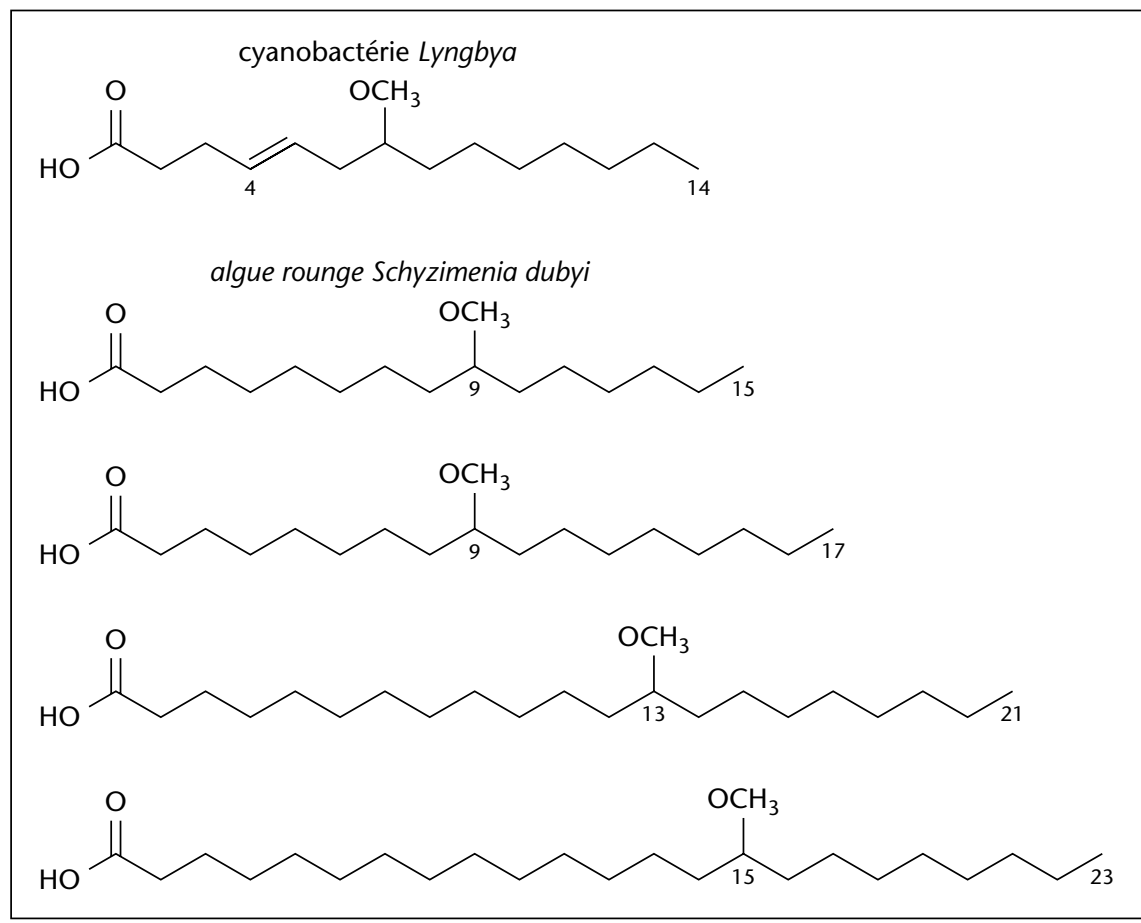

Figure 13. Autres acides gras méthoxylés marins.

des activités biologiques de ces AG marins originaux. Les investigations des nombreuses espèces des abysses et des milieux extrêmes pourraient ainsi conduire à de nouvelles découvertes.

\section{RÉFÉRENCES}

Ackman RG, Hooper SN. Non-methylene-interrupted fatty acids in lipids of shallow-water marine invertebrates: a comparison of two Molluscs (Littorina littorea and Lunatia triseriata) with the sand shrimp (Crangon septemspinosus). Comp Biochem Physiol B $1973 ; 46: 153-65$.

Ackman RG. Fatty acids. In : Ackman RG, ed. Marine biogenic lipids, fats, and oils, Vol. 1-2. Boca Raton, Florida, USA : CRC Press, 1989.

Andersson BA. Mass spectrometry of fatty acid pyrrolidides. Prog Chem Fats Other Lipids 1978 ; 16 : 279-308.

Ayanoglu E, Walkup RD, Sica D, Djerassi C. Phospholipid studies of marine organisms. III. New phospholipid fatty acids from Petrosia ficiformis. Lipids 1982 ; $17: 617-22$

Ayanoglu E, Komprobst JM, Aboud-Bichara A, Djerassi C. Phospholipid studies of marine organisms. IV. (2R,21Z)-2-methoxy-21-octacosenoic acid, the first naturally occurring $\alpha$-methoxy acid from a phospholipid. Tetrahedron Lett 1983 ; 24 : 1111-4.

Ayanoglu E, Popov S, Komprobst JM, Aboud-Bichara A, Djerassi C. Phospholipid studies of marine organisms. V. New $\alpha$-methoxy acids from Higginsia tethyoides. Lipids 1983b ; 18 : 830-6.
Barnathan G. Non-methylene-interrupted fatty acids from marine invertebrates: occurrence, characterization and biological properties. Biochimie 2009; 91 : 671-8.

Barnathan G, Mirallès ], Gaydou EM, Boury-Esnault $\mathrm{N}$, Kornprobst JM. New phospholipid fatty acids from the marine sponge Cinachyrella alloclada. Lipids $1992 ; 27: 779-84$.

Barnathan G, Kornprobst JM. Sponge fatty acids 2. Identification of three new $C_{30}$ fatty acids in the marine sponge Trikentrion loeve Carter. Nat Prod Lett $1992 ; 1: 201-7$.

Barnathan $G$, Doumenq $P$, Mirallès JM, Debitus $C$, Levi $C$, Kornprobst JM. Sponge fatty acids 3. Occurrence of complete series of $\mathrm{n}-7$ monoenoic and iso-5, 9 dienoic long-chain fatty acids from the New Caledonian sponge Cinachyrella aff. schulzei. Lipids $1994 ; 29$ : 297-303.

Barnathan G, Kornprobst JM, Doumenq P, Mirallès J. New unsaturated long-chain fatty acids in the phospholipids from the Axinellidae sponges Trikentrion loeve and Pseudaxinella cf. lunaecharta. Lipids $1996 ; 31: 193-200$.

Barnathan G, Bourgougnon N, Kornprobst JM. New methoxy fatty acids isolated from the red alga Schyzimenia dubyi. Phytochemistry 1998 ; 47 : 761-5.

Barnathan G, Genin E, Nongonierma R, Al-Lihaibi S, Velosaotsy NE, Kornprobst JM. Phospholipid fatty acids and sterols of two Cinachyrella from Saudi Arabia Red Sea. Comparative study with Cinachyrella sponges species from other origins. Comp Biochem Physiol B 2003 ; 135 : 297-308.
Bergé JP, Barnathan G. Recent advances in fatty acids from lipids of marine organisms: molecular biodiversity, roles as biomarkers, biologically-active compounds and economical aspects. In: Le Gal Y, Ulber R (eds) Marine Biotechnology. Adv Biochem Eng Biotechnol, Springer, 2005; $96:$ 49-125.

Boon JJ, de Leeuw JW, vd Hoek G), Vosjan JH. Significance and taxonomic value of iso and anteiso monoenoic fatty acids and branched hydroxy acids in Desulfovibrio desulfuricans. I Bacteriol 1977; 129 : 1183-91.

Boon PI, Virtue P, Nichols PD. Microbial consortia in wetland sediments: a biomarker analysis of the effects of hydrological regime, vegetation and season on benthic microbes. Mar Freshw Res 1996; 47 : 27-41.

Bourguet-Kondracki ML, Rakotoarisao MT, Martin MT, Guyot M. Bioactive bromopolyacetylenes from the marine sponge Xestospongia testudinaria. Tetrahedron Lett $1992 ; 33$ : 225-6.

Carballeira NM, Maldonaldo ME. On the isolation of the new fatty acid 6,11-eicosadienoic (20:2) and related 6,11-dienoic acids from the sponge Euryspongia rosea. Lipids $1989 ; 24$ : 665-8.

Carballeira NM, Restituyo J. Identification of the new 11,15-icosadienoic acid and related acids in the sponge Amphimedon complanata. / Nat Prod 1991 ; $54: 315-7$.

Carballeira NM, Maldonado ME. 7-Methyl-8hexadecenoic acid: a novel fatty acid from the marine sponge Desmapsama anchorata. Lipids 1988 ; 23 : 690-3

Carballeira NM, Reyes ED. Novel very long chain fatty acids from the sponge Petrosia pellasarca. I Nat Prod 1990a ; $53: 836-40$.

Carballeira NM, Reyes ED. Identification of the new 23-methyl-5,9-pentacosadienoic acid in the sponge Cribochalina vasculum. Lipids 1990b ; $25:$ 69-71.

Carballeira NM, Shalabi F. Identification of naturally occurring trans-trans delta-5,9 fatty acids from the sponge Plakortis halichondroides. Lipids 1990c ; 25 : 835-40.

Carballeira NM, Anastacio E, Salva J, Ortega MJ. Identification of the new 10,15-eicosadienoic acid and related acids in the opistobranch Haminaea templadoi. / Nat Prod 1992 ; 55 : 1783-6.

Carballeira NM, Emiliano A. Novel brominated phospholipid fatty acids from the Caribbean sponge Agelas sp. Lipids $1993 ; 28$ : 763-6.

Carballeira NM, Shalabi F. Novel brominated phospholipid fatty acids from the Caribbean sponge Petrosia sp. I Nat Prod 1993 ; 56 : 739-46.

Carballeira NM, Shalabi F. Unusual lipids in the Caribbean sponges Amphimedon viridis and Desmapsamma anchorata. J Nat Prod 1994a ; 57 : 1152-9.

Carballeira NM, Medina JR. New $\Delta 5,9$ fatty acids in the phospholipids of the sea anemone Stoichactis heliantus. J Nat Prod 1994b ; 57 : 1688-95.

Carballeira NM, Reyes M. Identification of a new 6-bromo-5,9-eicosadienoic acid from the anemone Condylactis gigantea and the zoanthid Palythoa caribaeorum. J Nat Prod 1995 ; 58 : 1689-94. 
Carballeira NM, Sostre A, Rodriguez AD. Phospholipid fatty acid composition of Gorgonians of the genus Pseudopterogorgia: identification of tetracosapolyenoic acids. Comp Biochem Physiol B 1996; 113 : 781-3.

Carballeira NM, Sostre A, Rodriguez AD. Phospholipid fatty acid composition of Gorgonians of the genus Eunicea: further identification of tetracosapolyenoic acids. Comp Biochem Physiol B 1997a ; 118 : 257-60.

Carballeira NM, Reyes ED, Sostre A, Rodriguez AD, Rodriguez JL, Gonzales FA. Identification of the novel antimicrobial fatty acid (5Z, 9Z)-14-methyl5,9-pentadecadienoic acid in Eunicea succinea. I Nat Prod 1997b ; $60: 502-4$

Carballeira NM, Pagan M, Rodriguez AD. Identification and total synthesis of novel fatty acids from the Caribbean sponge Calyx podatypa. J Nat Prod 1998 ; $61: 1049-52$.

Carballeira NM, Colon R. The use of (S)(-)-1-(1-naphthyl)ethylamine as a resolving agent for a-methoxy fatty acids. Tetrahedron Asymmetry 1999 ; $10: 378-90$

Carballeira NM, Pagan M. New methoxylated fatty acids from the Caribbean sponge Callyspongia fallax. J Nat Prod $2001 ; 64$ : 620-3.

Carballeira NM, Cruz H, Hill CA, De Voss J], Garson M. Identification and total synthesis of novel fatty acids from the siphonarid limpet Siphonaria denticulata. J Nat Prod 2001 ; 64 : 1426-9.

Carballeira NM, Miranda C, Rodriguez AD. Phospholipid fatty acid composition of Gorgonia mariae and Gorgonia entalina. Comp Biochem Physiol B 2002; 131: 83-7.

Carballeira NM. New advances in the chemistry of methoxylated lipids. Prog Lipid Res 2002; 41 : 433-56 (Review).

Carballeira NM, Miranda C. The first total synthesis of the marine fatty acid ( \pm )-9-methoxypentadecanoic acid: a synthetic route towards mid-chain methoxylated fatty acids. Chem Phys Lipids 2003 ; 124 : 63-7.

Carballeira NM, Sanabria D, Oyola D. An improved synthesis for the (Z)-14-methyl-9-pentadecenoic acid and its topoisomerase I inhibitory activity. Arkivoc 2007a ; $8: 49-57$.

Carballeira NM, Montano N, Padilla LF. First total synthesis of (Z)-15-methyl-10-hexadecenoic acid and the (Z)-13-methyl-8-tetradecenoic acid. Chem Phys Lipids 2007b ; 145 : 37-44.

Carballeira NM. New advances in fatty acids as antimalarial, antimycobacterial and antifungal agents. Prog Lipid Res 2008 ; 47 : 50-61.

Carballeira NM, Montano N, Balaña-Fouce R, Fernández Prada C. First total synthesis and antiprotozoal activity of (Z)-17-methyl-13-octadecenoic acid, a new marine fatty acid from the sponge Polymastia penicillus. Chem Phys Lipids 2009 ; 161 : 38-43.

Christie WW, Brechany EY, Stefanov KL, Popov S. The fatty acids of the sponge Dysidea fragilis from the Black Sea. Lipids 1992 ; 27 : 640-4.
Christie WW, Brechany EY, Marekov KL, Stefanov KL, Andreev SN. The fatty acids of the sponge Hymeniacidon sanguinea from the Black Sea. Comp Biochem Physiol A 2004 ; 109 : 245-52.

Christie WW. The Lipid Library. www.lipidlibrary.co.uk (mise à jour : 9 juillet 2010).

Cimino G, de Giulio A, de Rosa S, de Stefano S, Sodano $G$. Further high molecular weight polyacetylenes from the sponge Petrosia ficiformis. I Nat Prod $1985 ; 48: 22-7$.

Cuvelier C, Cabaraux JF, Dufrasne I, Hornick JL, Istasse L. Acides gras : nomenclature et sources alimentaires. Ann Med Vet $2004 ; 148: 133-40$.

Dasgupta A, Ayanoglu E, Djerassi C. Phospholipid studies of marine organsms: new branched fatty acids from Strongylophora durissima. Lipids 1984 ; $19: 768-76$.

Denis C, Wielgosz-Collin G, Bretéché A, et al. New 17-methyl-13-octadecenoic and 3,16-docosadienoic acids from the sponge Polymastia penicillus. Lipids $2009 ; 44:$ : 655-63.

Dembitsky VM, Srebnik M. Natural halogenated fatty acids: their analogues and derivatives. Prog Lipid Res 2002 ; 41 : 315-67.

Dembitsky VM. Anticancer activity of natural and synthetic acetylenic lipids. Lipids 2006 ; 41 : 883924 (Review).

Djerassi C, Lam WK. Sponge phospholipids. Acc Chem Res $1991 ; 24: 69-75$.

Dobson G, Christie WW. Spectroscopy and spectrometry of lipids (Part 2) - Mass spectrometry of fatty acid derivatives. Eur / Lipid Sci Technol 2002; 104 : 36-43.

Dowling NJE, Nichols PD, White DC. Phospholipid fatty acid and infra-red spectroscopic analysis of a sulfate-reducing consortium. FEMS Microbiol Ecol $1988 ; 53: 325-33$.

Fang J, Comet PA, Brooks JM, Wade TL. Nonmethylene interrupted fatty acids of hydrocarbon seep mussels: occurrence and significance. Comp Biochem Physiol B 1993 ; 104 : 287-91.

Fu X, Abbas SA, Schmitz FJ, Vidavskyt I, et al. New acetylenic metabolites from the marine sponge Pellina triangulata. Tetrahedron $1997 ; 53$ : 799-814.

Fusetani N, Li H, Tamura K, Matsuhaga S. Antifungal brominated $\mathrm{C}_{18}$ acetylenic acids from the marine sponge, Petrosiavolcano Hoshino. Tetrahedron $1993 ; 49$ : 1203-10.

Garson M], Zimmermann MP, Battershill CN, Holden JL, Murphy PT. The distribution of brominated longchain fatty acids in sponge and symbiont cell types from the tropical marine sponge Amphimedon terpenensis. Lipids $1994 ; 29$ : 509-16.

Garrido JL, Medina I. Identification of minor fatty acids in mussels (Mytilus galloprovincialis) by GC-MS of their 2-alkenyl-4,4-dimethyloxazoline derivatives. Anal Chim Acta 2002 ; 465 : 409-16.

Guo YW, Gavagnin M, Trivellone E, Cimino G. Absolute stereochemistry of petroformynes, high molecular polyacetylenes from the marine sponge Petrosia ficiformis. Tetrahedron $1994 ;$ 50 : 13261-8.
Gunstone FD, Harwood JL, Padley FB. The Lipid Handbook, $2^{e}$ edition. Londres, Royaume-Uni : Chapman and Hall Publishers, 1994.

Hakansson H, Sundin P, Anderson T, et al. In vitro and in vivo toxicity of fractionated fish lipids, with particular regard of chlorinated organic compounds. Pharmacol Toxicol $1991 ; 69$ : 459-71.

Harvey DJ. Mass spectrometry of picolinyl and other nitrogen-containing derivatives of fatty acids. In: Christie WW (ed) Advances in Lipid Methodology. Vol. 1. Royaume-Uni: The Oily Press, Dundee, 1992.

Hirsch SC, Carmely S, Kashman Y. Brominated unsaturated acids from the marine sponge Xestospongia sp. Tetrahedron $1987 ; 43: 3257-61$.

Isaacs S, Kashman Y, Loya S, Hizi A, Loya Y. Petrosynol and petrosolic acid, two novel natural inhibitors of the reverse transcriptase of human immunodeficiency virus from Petrosia sp. Tetrahedron 1993; 49 : 10435-8.

Ishihara K, Murata M, Kaneniwa M, et al. Effect of tetracosahexaenoic acid on the content and release of histamine, and eicosanoid production in MC/9 mouse mast cell. Lipids 1998 ; 33 : 1107-14.

Jefferts $E$, Morales RW, Litchfield C. Occurrence of cis-5, cis-9-hexacosadienoic and cis-5, cis-9, cis19-hexacosatrienoic acids in the marine sponge Microciona prolifera. Lipids $1974 ; 9$ : 244-7.

Joseph JD, Fender DS. Non-methylene interrupted dienes in decapod crustaceans of the Southeast Atlantic Ocean. J Am Oil Chem Soc 1977 ; 54 : 145A146A.

Johns RB, Nichols PD, Perry G). Fatty acid components of nine species of molluscs of the littoral zone from Australian waters. Comp Biochem Physiol B $1980 ; 65$ : 207-14.

Kaneda T. Iso- and anteiso-fatty acids in bacteria: biosynthesis, function, and taxonomic significance. Microbiol Rev $1991 ; 55$ : 288-302.

Kaneda T. Fatty acids of the genus Bacillus: an example of branched-chain preference. Bacteriol Rev 1997 ; 41 : 391-418.

Kawasaki K, Nabeshima YI, Ishihara K, Kaneniwa M, Ooizumi T. High level of $6,9,12,15,18,21$ tetracosahexaenoic acid found in lipids of Ophiuroidea Ophiura sarsi Lütken. Fish Sci 2000 ; 66 : 614-5.

Kawashima H, Ohnishi M. Fatty acid composition of various tissue lipids in the marine bivalves, Megangulus venulosus and Megangulus zyonoensis, from coastal waters of Hokkaido, Northern Japan. J Oleo Sci 2003 ; 52 : 309-15.

Kawashima K, Ohnishi M. Identification of minor fatty acids and various nonmethylene-interrupted diene isomers in mantle, muscle, and viscera of the marine bivalve Megangulus zyonoensis. Lipids 2004 ; 39 : 265-71.

Kawashima K, Ohnishi M. Occurrence of novel nonmethylene-interrupted $C_{24}$ polyenoic fatty acids in female gonad lipids of the limpet Cellana grata. Biosci Biotechnol Biochem 2008 ; 43 : 559-67. 
Kerger BD, Nichols PD, Antworth CP, et al. Signature fatty acids in the polar lipids of acid-producing Thiobacillus ssp. Methoxy, cyclopropyl, $\alpha$-hydroxycyclopropyl and branched and normal monoenoic fatty acids. FEMS Microbiol Ecol 1986 ; 38 : 67-77.

Klingensmith JS. Distribution of methylene and nonmethylene-interrupted dienoic fatty acids in polar lipids and triacylglycerols of selected tissues of the hardshell clam (Mercenaria mercenaria). Lipids 1982 ; $17: 976-81$

Kornprobst JM. Encyclopedia of Marine Natural Products, Wiley-Blackwell, 2010, 3 vol.

Kornprobst JM, Barnathan G. Methoxy fatty acids in nature. Recent Res Dev in Lipids Res $1998 ; 2$ : 371-80.

Kraffe E, Soudant P, Marty Y. Fatty acids of serine, ethanolamine, and choline plasmalogens in some marine bivalves. Lipids $2004 ; 39$ : 59-66.

Lam WK, Hahn S, Ayanoglu E, Djerassi C. Phospholipid studies of marine organisms. 22. Structure and biosynthesis of a novel brominated fatty acid from a hymeniacidonid sponge. J Org Chem 1989; 54 : 3428-32.

Li Y, Ishibashi M, Sasaki T, Kabayashi J. New brominecontaining unsaturated fatty acid derivatives from the Okinawan marine sponge Xestospongia sp. J Chem Res (M) 1995 ; 901-21.

Lee HK, Lee DS, Lim J, Kim JS, Im KS, Jung JH. Topoisomerase I inhibitors from the Streptomyces sp. strain KM86-9B isolated from a marine sponge. Arch Pharm Res $1998 ; 21$ : 729-33.

Lee HS, Rho JR, Sim C], Shin J. New acetylenic acids from a sponge of the genus Stelletta. I Nat Prod $2003 ; 66: 566-8$.

Leray C. GERLI, The CyberLipid Center. www.cyberlipid.org/index.htm (mise à jour : 12 juillet 2010).

Litchfield C, Tyskiewicz J, Marcantonio EE, Noto G. 15,18,21,24-tricontatetraenoic and 15,18,21,24,27tricontapentaenoic acids: new $C_{30}$ fatty acids from the marine sponge Cliona celata. Lipids $1979 ; 14$ : 619-22.

Mansour MP, Volkman JK, Holdworth DG, Jackson $A E$, Blackburn SI. Very-long chain $\left(C_{28}\right)$ highly unsaturated fatty acids in marine dinoflagellates. Phytochemistry 1999a ; 50 : 541-8.

Mansour MP, Volkman JK, Jackson AE, Blackburn SI. The fatty acid and sterol composition of five marine dinoflagellates. J Phycol 1999b ; 35 : 710-20.

Mansour MP, Holdsworth DG, Forbes S, Macleod C, Volkman JK. High contents of 24:6(n-3) and 20:1(n13) fatty acids in the brittle star Amphiura elandiformis from Tasmanian coastal sediments. Biochem Syst Ecol $2005 ; 33: 659-74$.

Matsunaga S, Okada Y, Fusetani N, Van Soest RWM. An antimicrobial $C_{14}$ acetylenic acid from a marine sponge Oceanapia species. I Nat Prod 2000; 63 : 690-1.

Mesguiche V, Valls R, Piovetti L, Peiffer G. Characterization and synthesis of ( \pm )-7-methoxydodec-4(E)enoic acid, a novel fatty acid isolated from Lyngbya majuscula. Tetrahedron Lett 1999 ; 40 : 7473-6.
Minto RE, Blacklock BJ. Review. Biosynthesis and function of polyacetylenes and allied natural products. Prog Lipid Res 2008 ; 47 : 233-306.

Mirallès J, Barnathan G, Galonnier R, et al. New branched-chain fatty acids from the Senegalese gorgonian Leptogorgia piccola (white and yellow morphs). Lipids 1995 ; 30 : 459-66.

Morales RW, Litchield C. Incorporation of ${ }_{1-}^{14} \mathrm{C}$-acetate into $\mathrm{C}_{26}$ fatty acids of the marine sponge Microciona prolifera. Lipids 1977; 12: 570-6.

Mu H, Wesén C, Novák T, Sundin P, Skramstad J, Odham G. Enrichment of chlorinated fatty acids in fish lipids prior to analysis by capillary gas chromatography with electrolytic conductivity detection and mass spectrometry. / Chromatog A 1996; 731 : 225-36.

Mu H, Wesen C, Sundin P. Halogenated fatty acids: II. Methods of determination in lipids. Trends Anal Chem $1997 ; 16$ : 266-74.

Murphy K, Mooney BD, Nichols PD, Sinclair AJ. Lipid, fatty acid and sterol composition of New Zealand green lipped mussel (Perna canaliculus) and Tasmanian blue mussel (Mytilus edulis). Lipids 2002; 37 : 587-95.

Nechev J, Christie WW, Robain R, de Diego F, Popov $S$. Chemical composition of the sponge Hymeniacdon sanguinea from the Canary Islands. Comp Biochem Physiol A 2004 ; 137 : 365-74.

Nichols PD, Danaher KT, Koslow JA. Occurrence of high levels of tetracosahexaenoic acid in the jellyfish Aurelia sp. Lipids 2003 ; 38 : 1207-10.

Ould El Kebir MV, Barnathan G, Siau Y, Mirallès ], Gaydou EM. Fatty acid distribution in muscle, liver, and gonads of rays (Dasyatis marmorata, Rhinobatos cemiculus, and Rhinoptera marginata) from the East Tropical Atlantic Ocean. J Agric Food Chem $2003 ; 51$ : 1942-7.

Ould El Kebir MV, Barnathan G, Siau Y, Mirallès ], Gaydou EM. Fatty acids in selected tissues of three tropical rays including five non-methyleneinterrupted dienoic fatty acids. Lipids $2007 ; 42: 525-35$.

Paradis M, Ackman RG. Occurrence and chemical structure of nonmethylene-interrupted dienoic fatty acids in American oyster Crassostrea virginica. Lipids $1975 ; 10: 12-6$.

Paradis M, Ackman RG. Potential for employing the distribution of anomalous non-methylene-interrupted dienoic fatty acids in several marine invertebrates as part of food web studies. Lipids $1977 ; 12$ : 170-6.

Patil AD, Kokke WC, Cochran S, Francis TA, Tomszek T, Westley JW. Brominated polyacetylenic acids from the marine sponge Xestospongia muta: inhibitors of HIV protease. I Nat Prod $1992 ; 55$ : 1170-7.

Pham NB, Butler MS, Hooper JNA, Moni RW, Quinn RJ. Isolation of xestosterol esters of brominated acetylenic fatty acids from the marine sponge Xestospongia testudinaria. / Nat Prod 1999 ; 62 : 1439-42.
Quinn RJ, Tucker DJ. A brominated bisacetylenic acid from the marine sponge Xestospongia testudinaria. Tetrahedron Lett $1985 ; 26$ : 1671-2.

Ratnayake WMN, Olsson B, Ackman RG. Novel branched-chain fatty acids in certain fish oils. Lipids $1989 ; 24$ : 630-7.

Reyes ED, Carballeira NM. A short synthesis of (Z)15-methylhexadec-11-enoic acid. Synthesis 1996: 693-4.

Ruiz N, Dubois N, Wielgosz-Collin G, et al. Lipid content and fatty acid composition of a marinederived Trichoderma longibrachiatum strain cultured by agar surface and submerged fermentations. Process Biochem $2007 ; 42: 676-80$.

Saito $H$. Unusual novel n-4 polyunsaturated fatty acids in cold-seep mussels (Bathymodiolus japonicus and Bathymodiolus platifrons), originating from symbiotic methanotrophic bacteria. / Chromatogr A $2008 ; 1200: 242-54$.

Sato D, Ando Y, Tsujimoto R, Kawasaki K. Identification of novel nonmethylene-interrupted fatty acids, $7 E, 13 E-20: 2,7 E, 13 E, 17 Z-20: 3,9 E, 15 E, 19 Z-22: 3$, and $4 Z, 9 E, 15 E, 19 Z-22: 4$, in Ophiuroidea (Brittle Star) lipids. Lipids $2001 ; 36: 1371-5$.

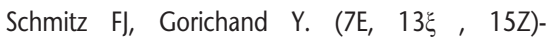
14,16-dibromo-7,13,15-hexadecatrien-5-ynoic acid. A novel dibromo acetylenic acid from the marine sponge Xestospongia muta. Tetrahedron Lett 1978 ; 19 : 3637-41.

Sörensen NA. Some naturally occurring acetylenic compounds. Proc Chem Soc 1961 : 98-110.

Takagi T, Kaneniwa M, Itabashi Y. Fatty acids in Crinoidea and Ophiuroidea: occurrence of allcis-6,9,12,15,18,21-tetracosahexaenoic acid. Lipids $1986 ; 21: 430-3$.

Tasdemir D, Topaloglu B, Perozzo R, et al. Marine natural products from the Turkish sponge Agelas oroides that inhibit the enoyl reductase from Plasmodium falciparum, Mycobacterium tuberculosis and Escherichia coli. Bioorg Med Chem 2007; 15: 6834-45.

Taylor J, Parkes JR. The cellular fatty acids of the sulfate-reducing bacteria, Desulfobacter species, Desulfobulbus species and Desulfovibrio desulfuricans. / Gen Microbiol 1983 ; 129 : 3303-9.

Tinsley LJ, Lowry RR. Bromine content of lipids of marine organisms. I Am Oil Chem Soc 1980; 57 : 31-3.

Trigari G, Pirini M, Pagliarani A, Manuzzi MP, Ventrella $\mathrm{V}$. High-levels of NMID fatty acids in molluscs. Ital J Biochem 2001 ; 50 : 41-6.

Vacelet J. Étude en microscopie électronique de l'association entre bactéries et spongiaires du genre Verongia (Dictyoceratia). J Microscopie Biol Cell 1975 ; $3: 271-88$.

Van Soest RWM, Fusetani N, Andersen RJ. Straightchain acetylenes as chemotaxonomic markers of the marine Haplosclerida. In : Watanabe Y, Fusetani N, eds. Sponge Sciences-Multidisciplinary Perspectives. Berlin, New York : Springer-Verlag, 1997. 
Ventrella V, Pirini M, Pagliarani A, Trombetti F, Manuzzi MP, Borgatti AR. Effect of temporal and geographical factors on fatty acid composition of Mytilus galloprovincialis from the Adriatic sea. Comp Biochem Physiol B $2008 ; 149$ : 241-50.

Vysotskii MV, Svetashev VI. Fatty acids of Heliopora coerulea and chemotaxonomic significance of tetracosapolyenoic acids in Coelenterates. Biochim Biophys Acta $1991 ; 1083$ : 161-5.

Walkup RD, Jamieson GC, Ratcliff MR, Djerassi C. Phospholipid studies of marine organisms: 2. Phospholipid-bound fatty acids and free sterols of the sponge Aplysina fistularis (Pallas) forma fulva (Verongia thiona). Isolation and structure elucidation of unprecedented branched fatty acids. Lipids 1981 ; $16: 631-46$.
Wesen C, Mu H, Sundin P, Froyen P, Skramstad J, Odham $\mathrm{G}$. Gas chromatographic-mass spectrometric identification of chlorinated octadecanoic acids in eel lipids. J Mass Spectrom 1995 ; 30 : 959-68.

White RH, Hager LP. Occurrence of fatty acid chlorohydrins in jellyfish lipids. Biochemistry $1977 ; 16$ : 4944-8.

Wijekoon WMD, Ayanoglu E, Djerassi C. Phospholipid studies of marine organisms 9 . New brominated demospongic acids from the phospholipids of two Petrosia species. Tetrahedron Lett 1984; 25: 3285-8.

Zakhartsev MV, Naumenko NV, Chelomin VP. Nonmethylene interrupted fatty acids in phospholipids of the membranes of the mussel Crenomytilus grayanus. Russ J Mar Biol 1998 ; 24 : 183-6.
Zhao Q, Lee SY, Hong J, et al. New acetylenic acids from the marine sponge Stelletta species. I Nat Prod $2003 ; 66: 408-11$.

Zhukova NV. Biosynthesis of non-methylene-interrupted fatty acids from $\left[{ }^{14} \mathrm{C}\right]$ acetate in molluscs. Biochim Biophys Acta $1986 ; 878$ : 131-3.

Zhukova NV. The pathway of the biosynthesis of non-methylene-interrupted dienoic fatty acids in molluscs. Comp Biochem Physiol B 1991; 100 : 801-4.

Zhukova NV, Svetashev VI. Non-methylene-interrupted dienoic fatty acids in molluscs from the Sea of Japan. Comp Biochem Phys B 1986 ; 83 : 643-6.

Zhukova NV. Lipid classes and fatty acid composition of the tropical Nudibranch mollusks Chromodoris sp. and Phyllidia coelestis. Lipids 2007 ; 42 : 1169-75. 